\title{
A computational modeling on transient heat and fluid flow through a curved duct of large aspect ratio with centrifugal instability
}

\author{
Shamsun Naher Dolon ${ }^{1}$, Mohammad Sanjeed Hasan ${ }^{2}$, Giulio Lorenzini ${ }^{3, a}$, \\ Rabindra Nath Mondal ${ }^{1}$ \\ ${ }^{1}$ Department of Mathematics, Jagannath University, Dhaka 1100, Bangladesh \\ 2 Department of Mathematics, Bangabandhu Sheikh Mujibur Rahman Science and Technology \\ University, Gopalganj 8100, Bangladesh \\ 3 Department of Engineering and Architecture, University of Parma, Parco Area delle Scienze 181/A, \\ 43124 Parma, Italy
}

Received: 3 February 2021 / Accepted: 17 March 2021

(C) The Author(s) 2021

\begin{abstract}
Due to remarkable applications of the curved ducts in engineering fields, scientists have paid much attention to invent new characteristics of curved-duct flow in mechanical systems. In the ongoing study, a computational modeling of fluid flow and energy distribution through a curved rectangular duct of large aspect ratio is presented. Governing equations are enumerated by using a spectral-based numerical technique together with the function expansion and collocation method. The main purpose of the paper is to analyze the effect of centrifugal force in the flow transition as well as heat transfer in the fluid. The investigations are performed for the aspect ratio, $\mathrm{Ar}=4$; the curvature ratio, $\delta=0.5$; the Grashof number, $\mathrm{Gr}=1000$; and varying the Dean number, $0<\mathrm{Dn} \leq 1000$. It is found that various types of flow regimes including steady-state and irregular oscillations occur as Dn is increased. To well understand the characteristics of the flow phase spaces and power spectrum of the solutions are performed. Next, pattern variations of axial and secondary flow velocity with isotherms are illustrated for different Dn's. It is revealed that the flow velocity and the isotherms are significantly influenced by the duct curvature and the aspect ratio. Convective heat transfer and temperature gradients are calculated which explores that the fluids are diversified due to centrifugal instability, and as a consequence the overall heat transfer is enhanced significantly in the curved duct.
\end{abstract}

\section{Introduction}

Investigation of centrifugal instability and heat convection in a curved duct is a fundamental and classic topic to the researchers in recent times due to noteworthy uses in chemical, biochemical and mechanical engineering such as in solar collectors, ventilating, chemical reactors, etc., and scholars are always alert and aware of using these two forces to construct curved-duct-based mechanical devices. The word "centrifugal force" was first used by Dean [1] after his pioneer work in 1927 . He observed that centrifugal force is affected by the duct

\footnotetext{
a e-mail: giulio.lorenzini@unipr.it (corresponding author)
} 
curvature, and under a critical flow condition the two-vortex secondary flow changes into fourvortex. The newly two vortices are designated in their study as Dean vortex. In the literature, there are numerous studies on fluid flows through different types of ducts; some of them may be mentioned by Mondal [2] (curved square duct (CSD) and CRD), Chandratilleke et al. [3-5] (elliptical and rectangular duct), Ahmadpour and Akhavan-Behabadi [6] (U-shaped duct), Abu-Hamdeh et al. [7] (helical tube), Pourhedayat et al. [8] (cylindrical tube) and Umavathi and Bég [9] (straight duct) for some outstanding works on this field.

In general, when fluid flows through a curved duct there arise some important features that are observed very carefully by the researchers. Yanase et al. $[10,11]$ reported a spectralbased numerical study to examine bifurcation formation through a CRD with linear stability analysis that applying Grashof numbers or not. A comprehensive study on bifurcation and flow transition including HT in the curved duct has been conducted by Mondal et al. [12] and Hasan et al. [13]. Two different types of bifurcations have been obtained by them. For the case of trembling solution, they demonstrated that when a critical point and bifurcation occurs, the steady-state flow changes into others regular and irregular flows. Very recently, Tanweer et al. [14] considered three-dimensional geometry to calculate unsteady behavior with respect to drag and lift coefficient. To show the unsteady behavior properly they discussed the power spectrum density. Hashemi et al. [15] addressed velocity fluctuation with flow transition and the consequences of bifurcation in turbulent flow through the curved tubing. Alonso et al. [16] explained the variation of flow patterns in respect of time for a vertical cylinder. Time-average streamline variations in the ingoing-outgoing channel has been investigated by Kurtulmuş et al. [17]. Islam et al. [18] narrated the time-dependent solution structure for rotating CRD of $\mathrm{Ar}=2$ and observed the change of instable characteristics for two different Dean numbers. Ray et al. [19] discussed the change of secondary vortex in the periodic and irregular oscillations for time advancement flow where they considered that the heating lower wall and the cooling top wall. Zhou et al. [20] described the vibration of the flow through an elliptical cylinder showing the regular and irregular vibration by evaluating phase spaces. Zhang et al. [21] compared the dynamical responses in terms of HT for a rotating circular cylinder and an enclosure with different velocities. A correlation between bifurcation structure and unsteady behavior of the 2-D flow activity in a revolving and stationary duct has been disclosed by Hasan et al. [22, 23]. Yanase et al. [24] illustrated the time-independent flow characteristics for an extensive range of $\mathrm{Ar}, 1 \leq \mathrm{Ar} \leq 12$ through a non-rotating curved duct. In their analysis, they did not explain the modes of regular and irregular oscillation by obtaining phase space or power spectrum. Liu and Wang [25] elucidated the bifurcation structure variation for changing the grid points and then detected the unsteady behavior for lager aspect ratio of the curved duct. Mondal et al. [26] narrated unsteady flow characteristics for the heated side-wall of the curved duct for different pressure gradient parameters. To interpret the characteristics of periodic, multi-periodic and chaotic oscillation, phase spaces were further calculated in their study. In the above studies, scholars showed time-history analysis for an extensive domain of the $\mathrm{Dn}, \mathrm{Nu}$, Re etc.; and only one tool phase-space and power-spectrum was evaluated for justifying the characteristics of periodic and irregular oscillation. Even the papers did not illustrate the effect of the parameters and the duct size elaborately. The effort of this paper is to meet up the lacking informations by defining flow transformations involving centrifugal instability, duct aspect ratio and heating-induced buoyancy energy.

Flow velocity and isotherm elucidate fluid mixing with centrifugal and aspect ratio effects in the enhancement of heat which is widely used in biomedical engineering and pharmaceutical industry due to the practical-life applications such as blood flow through the complex non-dichotomously curved network of blood vessels. Chandratilleke et al. [3-5] performed a 
three-dimensional (3D) computational fluid dynamics (CFD) investigation utilizing helicity function that illustrates additional vortex configuration and advection properties in the flow through a rectangular and elliptical tube. Raza et al. [27] elucidated the shape effects of nanofluid in three different coordinate systems when the fluid flow passes through the channel. Umavathi et al. [28] illustrated the patterns of unsteady velocity and isotherm contours through the horizontal channels. Akram and Afzal [29] tried to expose the two-dimensional flow velocities and isotherms through a uniform channel. Mondal et al. [30, 31] performed transient testing of fluid flow in the rectangular/square channel and revealed that secondary flow contributes to heat generation. Norouzi et al. [32] conducted heat transport of viscoelastic fluids in a square geometry treating certain heat flux. In the succeeding paper, Norouzi and Biglari [33] obtained flow pattern variation in a curved rectangular duct by applying the perturbation technique analytically. Wu et al. [34] employed spectral scheme for the curved duct flow. The computational study analyzed the secondary flow pattern by introducing an azimuthal pressure at the outer surface of the wall. Nowruzi et al. [35] conducted energy gradient method to visualize the stream velocity contours for various Dean numbers and curvature ratios at $180^{\circ}$ inlets. They validated their results with the experimental outcome and CFD output, respectively. Abu-Hamdeh et al. [7] chose $k-\in$ model to depict the contours of flow velocity and isotherm in the helically coiled tube for high Reynolds number. Kushawaha et al. [36] adopted finite difference and successive over-relaxation method to demonstrate the five different cases of flow characteristics through the rectangular enclosure with changing the Prandtl number. Umavathi and Bég [9] tried to show the three-dimensional contours of secondary flow and explained the characteristics of two different fluids both theoretically and experimentally. Hasan et al. [37] and Sultana et al. [38] established a relation between the axial and secondary velocity and the isotherms for the curved duct flow. They alongside obtained that flow velocities are stronger in periodic, multi-periodic and chaotic flows than the steady-state flow. But their study was unable to show the structural change when the aspect ratio is increased. Liu et al. [39] drove PIV and POD approach to obtain the flow behavior experimentally through the U-bend duct. Influences on the vortices in the shape of the helical rectangular tube have been studied by Zhang et al. [40] experimentally. They further demonstrated the flow velocity by drawing vector plots. Bibin and Jayakumar [41] investigated the hydraulic flow behavior along the duct center. Zhang et al. [42] proposed a three-dimensional model to conduct the flow patterns in periodic oscillation at several inlets. Li et al. [43, 44] numerically and experimentally displayed the two- and three-dimensional contours of secondary flow to discuss the hydrodynamic instability and the aspect ratio effect for curved ducts. In the above cited studies, the authors did not disclose the connection between axial and secondary velocity and isotherms for a curved rectangular duct flow. But it is very important to establish a relationship between them. The ongoing paper is an endeavor to reduce this gap.

It is important that calculating the heat transfer through a curved duct is an important phenomenon because it is widely applied in metallic industry, chemical components, nuclear power engineering, petroleum engineering, aerospace engineering, centrifugal pumps, etc. Riyi et al. [45] numerically and experimentally illustrated the overall heat transfer through the eccentric annular in the cooled and heated sidewalls separately. Pourhedayat et al. [8] imposed triangular winglets in cylindrical tubes to observe the enhancement of heat transfer through the tubes. Zhang et al. [46] explained the heat transfer for changing the Reynolds number, temperature ratios and rotational numbers through a rotating channel by considering turbulence correction and $k-\omega$ model. Benzenine et al. [47] explored the HT phenomena three-dimensionally in a curved channel of large aspect ratio to reduce the manufacturing cost by inputting low-pressure drops and getting high temperatures. Chamkha et al. [48] measured 
the $\mathrm{Nu}$ for various parameters such as rotation, radiation, Reynolds number and magnetic parameter, and then described how these parameters are affected by the spinning scheme. Dogonchi et al. [49] adopted finite element approach to compare the overall heat flow between the elliptical cylinder and enclosure in the presence of the Rayleigh number, Darcy number and aspect ratio. Shashikumar et al. [50] analytically demonstrated the electrical and thermal conductivity throughout the nano-channel where two different nanofluid particles were considered in their study. They further compared their analytical results with the numerical outcomes. Fallahnezhad and Nazif [51] narrated the forced convection and hydrodynamic instability through the channel where copper nanofluid is considered in their exploration. Selimefendigil et al. [52] employed Galerkin weighted residual technique to interpret the nanofluid forced convection in the branching channel. Baleanu et al. [53] analyzed the magnetic field and heat transfer effect among the SWC and MWC nanotubes. Yang et al. [54] employed defined-function and FLUENT software to enumerate the forced convective heat transfer through a vibrated cylinder. They further traced out the particle trajectories when the fluid particle is thermally insulated. Rajamohan et al. [55] experimentally expressed the convection and radiation of laminar flow through the channel whose lower wall was heated and upper wall cooled. Dolon et al. [56] sumptuously explained how heat transfer affects the secondary vortex structure as well as flow transition by showing the steam function and the isotherm. Hasan et al. [57] analyzed the influence of heat transformation in the steady and unsteady flow simultaneously. They noticed that the fluid is mixed more because of the vibration of the fluid particles and as a consequence heat is transferred substantially by the periodic and irregular oscillation. In the above study, however, scholars did not investigate the flow transition and heat transfer as well as flow behavior together and there are few works on these phenomena. The current paper, therefore, proposes to investigate the flow transition and heat transfer in a curved rectangular duct of large aspect ratio discussing the physics of the problem. Besides, centrifugal instability and aspect ratio effects in unsteady behavior, vortex structure and heat transfer with temperature gradients are illustrated elaborately. The present numerical work is validated with the experimental data as well.

\section{Flow model and governing equations}

The two-dimensional (2-D) geometric model of the flow through a CRD with necessary notations is illustrated in Fig. 1 where the fluid is considered to be laminar. These side walls are assumed to be differentially heated where the heating outside wall while the other three walls are adiabatic. As the flow geometry is considered in the cylindrical co-ordinate system, so $r, \theta$ and $y$ are assumed as the required axis. However, the conservation of mass, momentum and energy equations in cylindrical coordinate system are expressed as,

Conservation of mass equation:

$$
\frac{\partial v_{r}}{\partial r}+\frac{1}{r} \frac{\partial v_{\theta}}{\partial \theta}+\frac{\partial v_{y}}{\partial y}+\frac{v_{r}}{r}=0
$$

Momentum equations:

$$
\begin{gathered}
\frac{\partial v_{r}}{\partial t}+(v \cdot \nabla) v_{r}-\frac{v_{\theta}^{2}}{r}=-\frac{1}{\rho} \frac{\partial P}{\partial r}+v\left(\tilde{\Delta} v_{r}-\frac{v_{r}}{r^{2}}-\frac{2}{r^{2}} \frac{\partial v_{\theta}}{\partial \theta}\right) \\
\frac{\partial v_{\theta}}{\partial t}+(v \cdot \nabla) v_{\theta}+\frac{v_{r} v_{\theta}}{r}=-\frac{1}{\rho r} \frac{\partial P}{\partial \theta}+v\left(\tilde{\Delta} v_{\theta}-\frac{v_{\theta}}{r^{2}}+\frac{2}{r^{2}} \frac{\partial v_{r}}{\partial \theta}\right) \\
\frac{\partial v_{y}}{\partial t}+(v \cdot \nabla) v_{y}=-\frac{1}{\rho} \frac{\partial P}{\partial y}+v \tilde{\Delta} v_{r}+\beta g T
\end{gathered}
$$




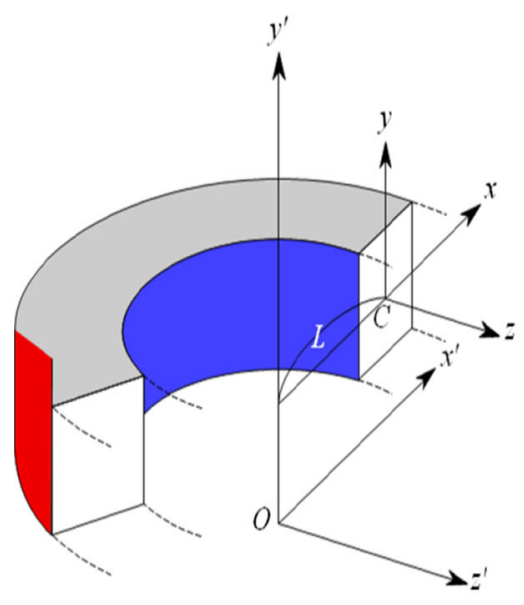

(a)

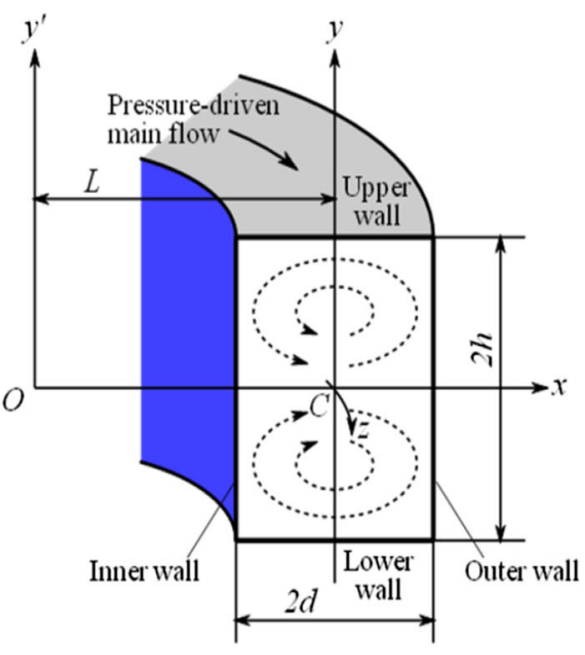

(b)

Fig. 1 a The physical model, b cross-sectional view of CRD

Energy equation:

$$
\frac{\partial T}{\partial t}+(v \cdot \nabla) T=\kappa \tilde{\Delta} T
$$

Here, $v \cdot \nabla=v_{r} \frac{\partial}{\partial r}+\frac{v_{\theta}}{r} \frac{\partial}{\partial \theta}+v_{y} \frac{\partial}{\partial y}, \tilde{\Delta}=\frac{\partial^{2}}{\partial r^{2}}+\frac{1}{r} \frac{\partial}{\partial r}+\frac{1}{r^{2}} \frac{\partial^{2}}{\partial \theta^{2}}+\frac{\partial^{2}}{\partial y^{2}}$.

Here, $\kappa$; coefficient of thermal conductivity, $g$; gravitational acceleration, $v$; kinematic viscosity and $\rho$; density respectively. Now, Eqs. (1) to (5) are non-dimensionalized by the following assumptions:

$$
\begin{aligned}
& r=L+\mathrm{d} x^{\prime}, \quad y=h y^{\prime}, \quad L \theta=-\mathrm{d} z^{\prime}, T=T^{\prime} \Delta T, \quad v_{r}=v_{x}=U_{0} u^{\prime}, v_{y}=v_{y}=U_{0} v^{\prime}, \\
& v_{\theta}=-v_{z}=U_{0} w^{\prime}, \rho=\rho_{0} U_{0}^{2} P^{\prime}, G=-\frac{\partial P}{\partial z^{\prime}}, \in=\sqrt{\frac{2 d}{L}}=\sqrt{2 \delta}, \delta=\frac{d}{L}, \operatorname{Ar}=\frac{4 h}{d}
\end{aligned}
$$

where $G$; pressure gradient parameter which calculates the rate of pressure with respect to the particular axis; $\delta$; curvature and Ar; aspect ratio of the duct. In the present paper, the cross section is considered as rectangular. Since the fluid passes through consistently in the stream-wise direction, so $\frac{\partial p^{\prime}}{\partial z^{\prime}}=0$.

The transformed Eqs. (1) to (5) can be written as,

Conservation of mass equation:

$$
\frac{\partial u^{\prime}}{\partial x^{\prime}}+\frac{\partial v^{\prime}}{\partial y^{\prime}}+\frac{\delta u^{\prime}}{r^{\prime}}+\frac{1}{r^{\prime}} \frac{\partial w^{\prime}}{\partial z^{\prime}}=0
$$

Momentum equations:

$$
\begin{gathered}
\frac{\partial u^{\prime}}{\partial t^{\prime}}+\left(v^{\prime} \cdot \nabla^{\prime}\right) u^{\prime}-\frac{1}{2} \epsilon^{2} \frac{w^{\prime 2}}{r^{\prime}}=-\frac{\partial P^{\prime}}{\partial x^{\prime}}+\frac{\epsilon}{\mathrm{Dn}}\left(\tilde{\Delta}_{2}^{\prime} u^{\prime}-\frac{\delta^{2} u^{\prime}}{r^{\prime 2}}\right) \\
\frac{\partial v^{\prime}}{\partial t^{\prime}}+\left(v^{\prime} \cdot \nabla^{\prime}\right) v^{\prime}=-\frac{\partial P^{\prime}}{\partial y^{\prime}}+\frac{\epsilon}{\mathrm{Dn}} \tilde{\Delta}_{2}^{\prime} v^{\prime}+\frac{\beta g \Delta T l}{U_{0}^{2}} T^{\prime} \\
\frac{\partial w^{\prime}}{\partial t^{\prime}}+\left(v^{\prime} \cdot \nabla^{\prime}\right) w^{\prime}+\frac{1}{2} \epsilon^{2} \frac{u^{\prime} w^{\prime}}{r^{\prime}}=-\frac{G}{r^{\prime}}+\frac{\epsilon}{\mathrm{Dn}}\left(\tilde{\Delta}_{2}^{\prime} w^{\prime}-\frac{\delta^{2} u^{\prime}}{r^{\prime 2}}\right)
\end{gathered}
$$


Energy equation:

$$
\frac{\partial T^{\prime}}{\partial t^{\prime}}+\left(v^{\prime} \cdot \nabla^{\prime}\right) T^{\prime}=\frac{\kappa}{\mathrm{d} U_{0}} \tilde{\Delta}_{2}^{\prime} T^{\prime}
$$

where $\left(v^{\prime} \cdot \nabla^{\prime}\right)=u^{\prime} \frac{\partial}{\partial x^{\prime}}+v^{\prime} \frac{\partial}{\partial y^{\prime}}, r^{\prime}=1+\delta x^{\prime}$ and $\tilde{\Delta}_{2}^{\prime}=\frac{\partial^{2}}{\partial x^{\prime 2}}+\frac{\partial}{\partial y^{\prime 2}}+\frac{\delta}{r^{\prime}} \frac{\partial}{\partial x^{\prime}}$.

The vorticity vector and stream functions have the following form, respectively,

$$
\begin{gathered}
\Omega^{\prime}=\frac{\partial v^{\prime}}{\partial x^{\prime}}-\frac{\partial u^{\prime}}{\partial y^{\prime}}=-\frac{1}{r^{\prime}}\left(\frac{\partial^{2}}{\partial x^{\prime 2}}+\frac{\partial^{2}}{\partial y^{\prime 2}}-\frac{\delta}{r^{\prime}} \frac{\partial}{\partial x^{\prime}}\right) \\
u^{\prime}=\frac{1}{r^{\prime}} \frac{\partial \psi^{\prime}}{\partial y^{\prime}} \text { and } v^{\prime}=-\frac{1}{r^{\prime}} \frac{\partial \psi^{\prime}}{\partial x^{\prime}}
\end{gathered}
$$

Now $\frac{\partial}{\partial x^{\prime}}(8)-\frac{\partial}{\partial y^{\prime}}(9)$, we obtain the equation,

$$
\frac{\partial \Omega^{\prime}}{\partial t^{\prime}}+\left(u^{\prime} \frac{\partial}{\partial x^{\prime}}+v^{\prime} \frac{\partial}{\partial y^{\prime}}\right) \Omega^{\prime}-\frac{\delta u^{\prime}}{r^{\prime}} \Omega^{\prime}+\frac{\epsilon^{2} w^{\prime}}{r^{\prime}} \frac{\partial w^{\prime}}{\partial y^{\prime}}=\frac{\epsilon}{\operatorname{Dn}}\left(\tilde{\Delta}_{2}^{\prime}-\frac{\delta^{2}}{r^{\prime}}\right) \Omega^{\prime}+\frac{\beta g \Delta T \operatorname{Ar}}{U_{0}^{2}} \frac{\partial T^{\prime}}{\partial x^{\prime}}
$$

Then, modified basic equations for $\omega, \eta$, and $\xi$ as follows (eliminating $\left(^{\prime}\right)$ sign)

$$
\begin{aligned}
& r \frac{\partial \omega}{\partial t}+\frac{1}{4} \frac{\partial(\omega, \eta)}{\partial(x, y)}-\mathrm{Dn}+\frac{\delta^{2} \omega}{r}=r \Delta_{2} \omega-\frac{\delta}{4 r} \frac{\partial \eta}{\partial y} \omega+\delta \frac{\partial \omega}{\partial x} \\
& \left(\Delta_{2}-\frac{\delta}{r} \frac{\partial}{\partial x}\right) \frac{\partial \eta}{\partial t}=-\frac{1}{4 r} \frac{\partial\left(\Delta_{2} \eta, \eta\right)}{\partial(x, y)}+\frac{\delta}{r^{2}}\left[3 \delta \frac{\partial^{2} \eta}{\partial x^{2}}-\frac{3 \delta^{2}}{r} \frac{\partial \eta}{\partial x}\right] \\
& +\frac{\delta}{4 r^{2}}\left[\frac{\partial \eta}{\partial y}\left(2 \Delta_{2} \eta-\frac{3 \delta}{r} \frac{\partial \eta}{\partial x}+\frac{\partial^{2} \eta}{\partial x^{2}}\right)-\frac{\partial \eta}{\partial x} \frac{\partial^{2} \eta}{\partial x \partial y}\right] \\
& -\frac{2 \delta}{r} \frac{\partial}{\partial x} \Delta_{2} \eta+\frac{1}{4} \omega \frac{\partial \omega}{\partial y}+\Delta_{2}^{2} \eta-\operatorname{Gr} \times r \frac{\partial \xi}{\partial x} \\
& \frac{\partial \xi}{\partial t}+\frac{1}{4 r} \frac{\partial(\xi, \eta)}{\partial(x, y)}=\frac{1}{\operatorname{Pr}}\left(\Delta_{2} \xi+\frac{\delta}{r} \frac{\partial \xi}{\partial x}\right)
\end{aligned}
$$

where $\Delta_{2}=\frac{\partial^{2}}{\partial x^{2}}+\frac{1}{16} \frac{\partial^{2}}{\partial y^{2}}, r=1+\delta x$ and $\frac{\partial(f, g)}{\partial(x, y)}=\frac{\partial f}{\partial x} \frac{\partial g}{\partial y}-\frac{\partial f}{\partial y} \frac{\partial g}{\partial x}$.

The dimensionless parameters, Dean number; Dn Grashof number; Gr and Prandtl number; Pr are defined as:

$$
\operatorname{Dn}=\frac{G d^{3}}{\mu \nu} \sqrt{\frac{2 d}{L}}=\frac{G d^{3}}{\mu \nu} \sqrt{2 \delta}, \text { Gr }=\frac{\beta g \Delta T d^{3}}{\nu^{2}}, \operatorname{Pr}=\frac{\nu}{\kappa}
$$

The bounder conditions of the axial velocity $(\omega)$ and the sectional stream velocity $(\eta)$ are,

$$
\begin{aligned}
& \omega=\eta=\frac{\partial \eta}{\partial x}=0, \quad x= \pm 1, \quad y=y \\
& \omega=\eta=\frac{\partial \eta}{\partial y}=0, \quad x=x, y= \pm 1
\end{aligned}
$$

and the conducting boundary conditions for isotherms $(\xi)$ are taken as,

$$
\xi(1, y)=y, \xi(-1, y)=y, \xi(x, 1)=x, \xi(x,-1)=x
$$

In this study, water is considered as the working fluid $(\operatorname{Pr}=7.0)$; the Grashof number $(\mathrm{Gr})$ and the aspect ratio (Ar) are fixed as $\mathrm{Gr}=1000$ and 4, respectively, and the Dean number (Dn) varies $(0<\mathrm{Dn} \leq 1000)$. 


\section{Numerical calculations}

\subsection{Method of numerical design}

To obtain numerical solutions of Eqs. (14)-(16), spectral scheme is applied. According to the principle of this scheme, the variables are expanded in a series of functions consisting of the Chebyshev polynomials. That is, the functions expansion of $\varphi_{n}(x)$ and $\eta_{n}(x)$ are articulated as

$$
\begin{aligned}
& \varphi_{n}(x)=\left(1-x^{2}\right) F_{n}(x), \\
& \eta_{n}(x)=\left(1-x^{2}\right)^{2} F_{n}(x)
\end{aligned}
$$

where $F_{n}(x)=\cos \left(n \cos ^{-1}(x)\right)$ is the $n$ th-order Chebyshev polynomial. $\omega(x, y, t), \eta$ $(x, y, t)$ and $\xi(x, y, t)$ are stated $\varphi_{n}(x)$ and $\eta_{n}(x)$ as

$$
\begin{aligned}
& \omega(x, y, t)=\sum_{m=0}^{M} \sum_{n=0}^{N} \omega_{m n}(t) \phi_{m}(x) \phi_{n}(y) \\
& \eta(x, y, t)=\sum_{m=0}^{M} \sum_{n=0}^{N} \eta_{m n}(t) \eta_{m}(x) \eta_{n}(y) \\
& \xi(x, y, t)=\sum_{m=0}^{M} \sum_{n=0}^{N} \xi_{m n}(t) \varphi_{m}(x) \varphi_{n}(y)+x
\end{aligned}
$$

where $M$ and $N$ represent the truncation numbers in the $x$ - and $y$-directions, respectively, and $\omega_{m n}, \eta_{m n}$ and $\xi_{m n}$ are the coefficients of expansion.

To achieve time-dependent solutions, the expansion series (20) with coefficients $\omega_{m n}(t)$, $\eta_{m n}(t)$ and $\xi_{m n}(t)$ are converted into the basic Eqs. (14)-(16) abide by applying the CrankNicolson, Adams-Bashforth and the collocation method which are not shown here for brevity. Details of this method are available in Gottlieb and Orszag [58] and in Mondal [2].

\subsection{Resistance coefficient}

The resistance coefficient $\lambda$, another name the hydraulic resistance coefficient, designating the quantity of the flow state is stated as

$$
\frac{\bar{p}_{1}-\bar{p}_{2}}{\Delta_{\bar{z}}}=\frac{\lambda}{\bar{d}_{h}} \frac{1}{2} \rho\langle\bar{\sigma}\rangle^{2}
$$

The mean axial velocity $\langle\bar{\sigma}\rangle$ is calculated by

$$
\langle\bar{\sigma}\rangle=\frac{v}{4 \sqrt{2 \delta d}} \int_{-1}^{1} \mathrm{~d} x \int_{-1}^{1} \sigma(x, y, t) \mathrm{d} y
$$

$\lambda$ is related to the mean non-dimensional axial velocity as $\langle\omega\rangle=\sqrt{2 \delta d}\langle\bar{\sigma}\rangle / v$

$$
\lambda=\frac{32 \sqrt{2 \delta} \mathrm{Dn}}{5\langle\omega\rangle^{2}}
$$

\subsection{The Nusselt number $(\mathrm{Nu})$}

The ratio of convective to conductive HT throughout the duct is known as Nusselt number $(\mathrm{Nu})$. As the current study focuses on HT, there will be need to calculate two different types 
Table 1 Grid accuracy for $\mathrm{Dn}=$ $1000, \mathrm{Gr}=1000, \delta=0.5$ and $\mathrm{Ar}$ $=4$

\begin{tabular}{llll}
\hline$M$ & $N$ & $\lambda$ & $w(0,0)$ \\
\hline 14 & 50 & 0.366008633988 & 626.6143487316 \\
14 & 54 & 0.365991921591 & 626.6563173072 \\
16 & 56 & 0.366103444057 & 626.8017425746 \\
16 & 60 & 0.366108424501 & 626.7947121678 \\
16 & 64 & 0.366109012251 & 626.7939354819
\end{tabular}

of HT through the duct, HT in the steady and unsteady solution. The Nu for steady solution is enumerated by,

$$
\mathrm{Nu}=-\frac{d^{*}}{\Delta T^{*}}\left\langle\left.\frac{\partial T^{*}}{\partial x^{*}}\right|_{x=0}\right\rangle
$$

Here, \langle\rangle and $(*)$ represent the mean of HT from the fluid to the duct walls and the dimensionless quantities, respectively; $x$; distance of the walls; and $\Delta T$; difference of heat.

Now the Nusselt number for cooled and heated side walls are denoted by $\mathrm{Nu}_{\mathrm{c}}$ and $\mathrm{Nu}_{\mathrm{h}}$ for unsteady solution, respectively, and quantified by the following

$$
\mathrm{Nu}_{\tau_{c}}=\frac{1}{2} \int_{-1}^{1}\left\langle\left\langle\left.\frac{\partial T}{\partial x}\right|_{x=-1}\right\rangle\right\rangle \mathrm{d} y, \quad \mathrm{Nu}_{\tau_{h}}=\frac{1}{2} \int_{-1}^{1}\left\langle\left\langle\left.\frac{\partial T}{\partial x}\right|_{x=1}\right\rangle\right\rangle \mathrm{d} y
$$

Here $\tau$ is the average time-interval which is addressed by $\langle\langle\rangle\rangle$; and taken as one-period oscillation if periodic flow occurs; and chosen as a suitable time-interval if chaotic flow generates.

\subsection{Grid accuracy}

In this section, grid accuracy is computed for different grid numbers. Here $M$ and $N$ are addressed as the grid sizes. Here, five different grid sizes such as $14 \times 50,14 \times 54,16 \times$ $56,16 \times 60,16 \times 64$ are enumerated for fixed Dean and Grashof number, and curvature and aspect ratio as explored in Table 1 . It is seen that the findings are sufficiently accurate for increasing the grid numbers. In this study, $M=16$ and $N=60$ are considered to obtain the flow behavior as well as the heat transfer. Table 1 summarizes the parameters for the 5 test cases. A grid with dimensions of $20 \times 60$ is used.

\section{Results}

Here, three different types of investigations have been performed, namely transitional behavior of the time-dependent flow, convective heat transfer with temperature gradients and finally the numerical result is validated with experimental outcomes. All these phenomena are analyzed for aspect ratio (Ar) 4; curvature ratio $\delta=0.5$; the Grashof number $\mathrm{Gr}=1000$ over a wide range of the Dean number $0<\mathrm{Dn} \leq 1000$.

\subsection{Analysis of flow transitions}

To discern the flow transition of the present study, time-evolution calculations are performed for Dn $>0$ to Dn $=145$ and it is obtained that the unsteady solutions (US) give steady-state graph for these Dean numbers in time vs. Resistance coefficient plane. Figure $2 \mathrm{a}, \mathrm{b}$ depicts the 


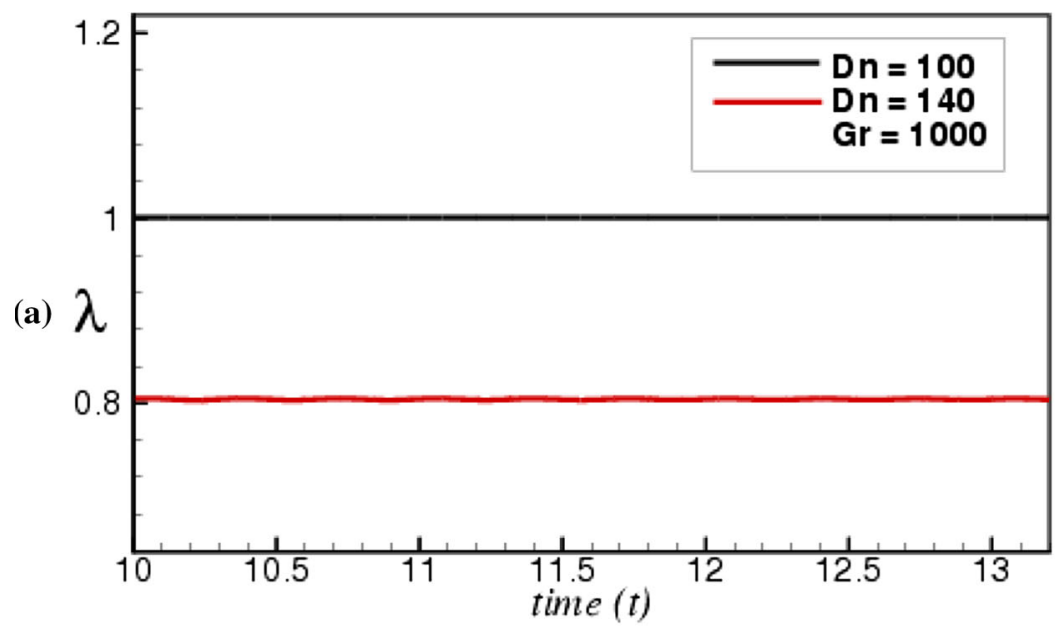

$\mathrm{Dn}=100$

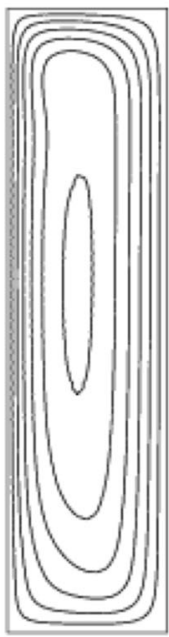

$\omega$

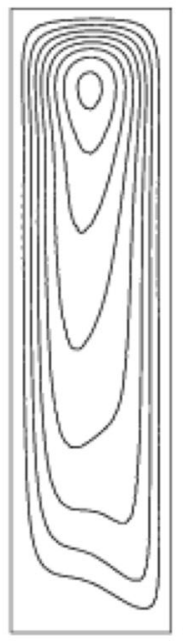

$\eta$

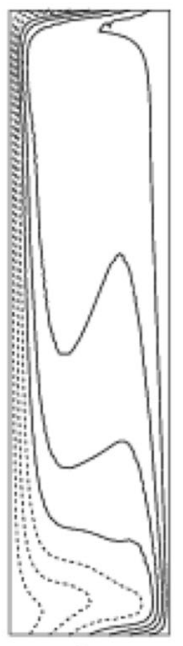

$\xi$

$\mathrm{Dn}=140$

(b)

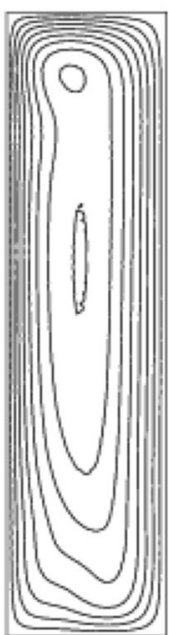

$\omega$

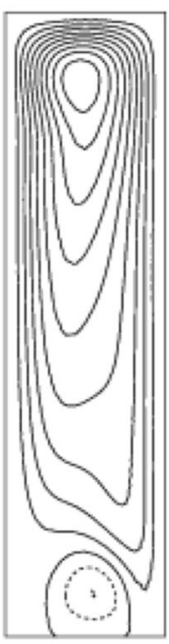

$\eta$

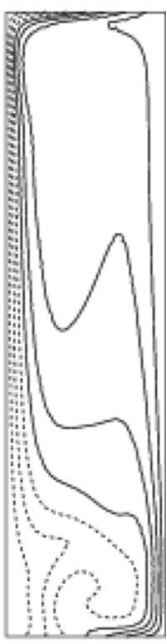

$\xi$

Fig. 2 a Unsteady solution, b axial (top) and secondary (middle) flow velocity and isotherms (bottom); for $\mathrm{Dn}=100$ and 140

US as well as flow patterns for $\mathrm{Dn}=100$ and $\mathrm{Dn}=140$, respectively. It is remarked in Fig. 2a that magnitude of the $\lambda$ is decreased for raising Dn and it is noticed that there arises singleand two-vortex solutions creating a robust link between the axial and secondary velocity. For $\mathrm{Dn}=100$, a single dumbbell has been generated in the middle of the axial flow, and as a result a single-vortex solution is created. On the contrary, at Dn $=140$, the two-vortex asymmetric secondary flow is produced at the duct upper and lower wall because a pair of dumbbells is created in the middle of the axial flow.

The steady-state flow converts into periodic flow if $\mathrm{Dn}$ is increased. The periodic oscillation begins at $\mathrm{Dn}=150$ and which continues up to $\mathrm{Dn}=180$. Figure $3 \mathrm{a}$ illustrates the periodic 


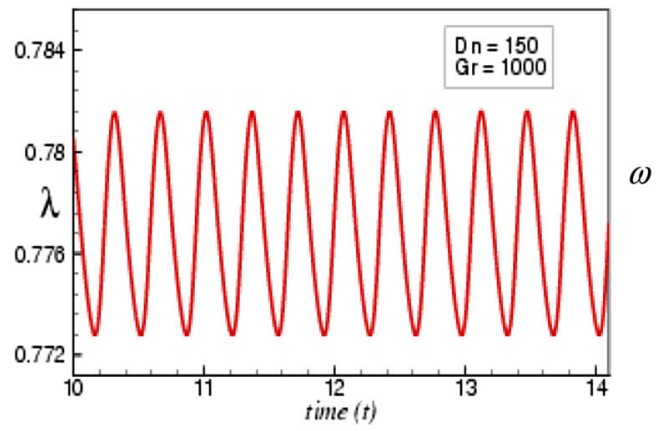

(a)
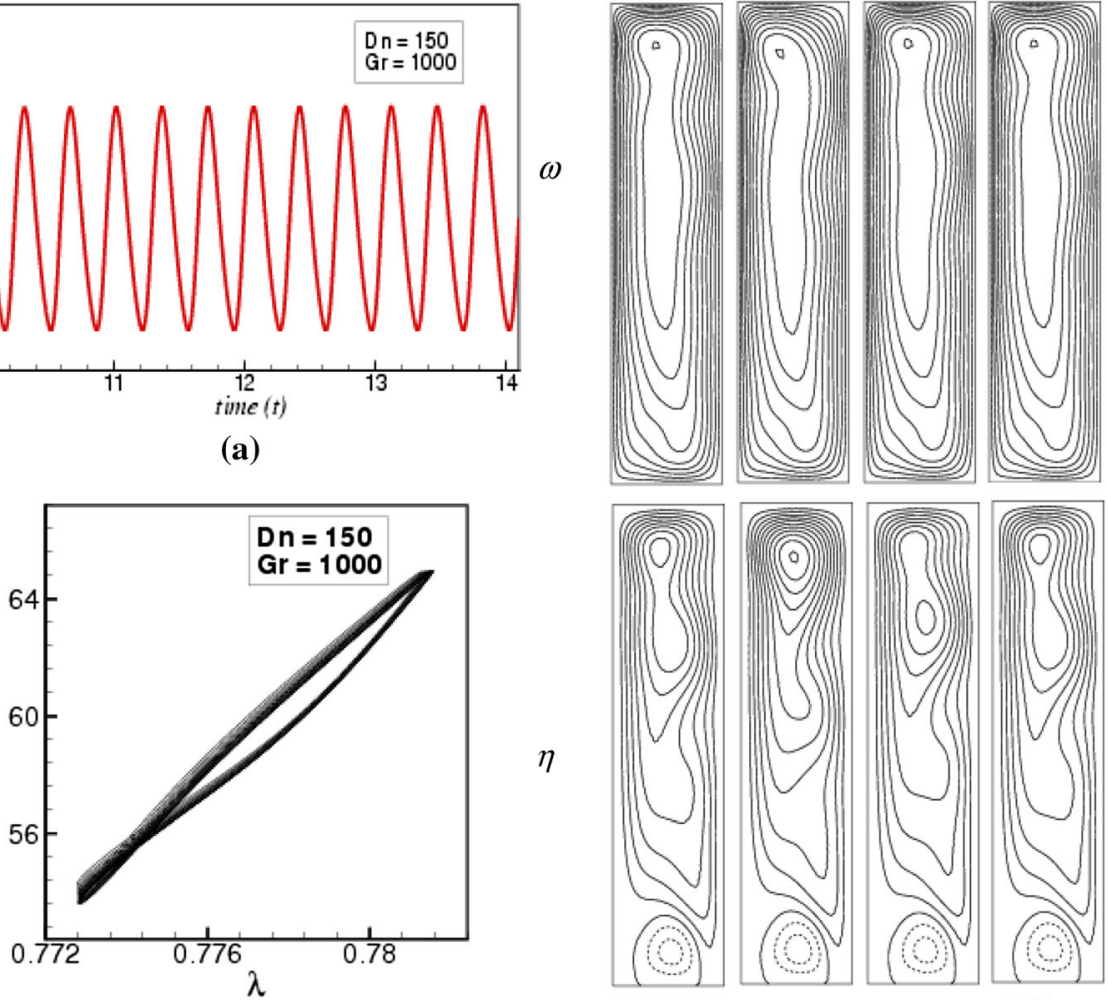

(b)

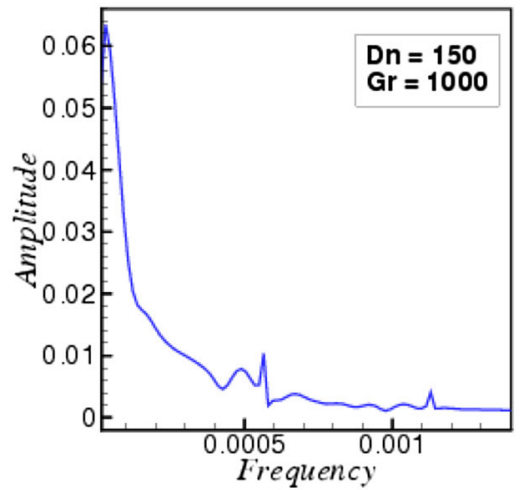

(c)
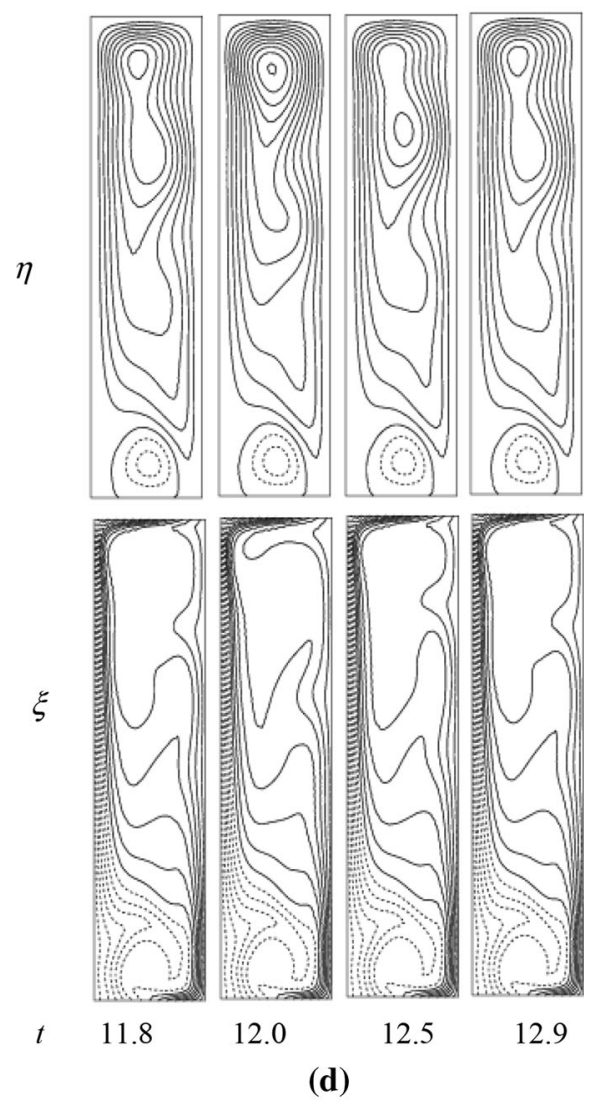

Fig. 3 a Unsteady solution, b phase space, $\mathbf{c}$ power spectrum, d axial (top) and secondary (middle) flow velocity and isotherms (bottom); for $\mathrm{Dn}=150$

oscillating flow at $\mathrm{Dn}=150$, and Fig. $3 \mathrm{~b}$, c represents the phase space and power spectrum to ensure more about the periodic flow. The phase space demonstrates the total area of path line of the stream function for a specific Dn. More clearly, the phase space is calculated by $\gamma=\iint \psi \mathrm{d} x \mathrm{~d} y$ and drawn in $\lambda-\gamma$ plane. As seen in Fig. $3 \mathrm{~b}$, the path lines of the stream velocity completed a single cycle and this velocity is limited to the specific orbit. On 
the other hand, the power spectrum illustrates in the Frequency vs. Amplitude plane which narrates about the ability of the oscillation of the flow behavior how strong or weak the flow is. It is obtained from Fig. $3 \mathrm{c}$ that the line spectrum as well as frequencies are less strong at 0.00125 than 0.000125 and this weak oscillation turns into a steady-state flow after crossing over 0.00125 , which certainly reported that the flow characteristics at Dn $=150$ are a periodic oscillating solution. Contours of axial flow, two-vortex asymmetric secondary flow and isotherms are shown in Fig. $3 \mathrm{~d}$ for $\mathrm{Dn}=150$.

The study shows that if Dn is increased a little, the flow fluctuations are significantly changed. It is obtained that multi-periodic oscillation occurs from $\mathrm{Dn}=190$ to $\mathrm{Dn}=220$ as revealed in Figs. 4a and 5a for $\mathrm{Dn}=190$ and $\mathrm{Dn}=220$, respectively. It is apparently seen that Fig. 4a shows the periodic oscillation. For this reason, phase space and power spectrum density are calculated and plotted in Fig. 4b, c consecutively. Phase space demonstrates that the path lines create a pair of orbits and the power spectrum describes that the oscillation of line spectrum and the frequencies become weaker gradually after vibrating a small range in the frequency-amplitude plane. Figure 4a shows the multi-periodic oscillation, and specifically it is assumed that it is the initial stage of multi-periodic solution rather than periodic. Axial and secondary flow velocity and temperature profiles are plotted in Fig. 4d. It is observed that two- and three-vortex solutions are found at $\mathrm{Dn}=190$ and the density of isotherm is more for multi-periodic $(\mathrm{Dn}=190)$ flow than the periodic flow $(\mathrm{Dn}=150)$. Secondary flow demonstrates that from $t=16.30$ to $t=16.50$, the flow completes multiple cycles, i.e., the multi-periodic oscillation at $\mathrm{Dn}=190$ repeats after $20 \mathrm{~s}$ consecutively. On the other hand, Fig. 5a-c shows that the flow behavior is clearly multi-periodic without any doubt. The flow patterns and the isotherms are also exhibited in Fig. 5d where it is seen that the axial flows are pushed to the outer wall of the duct and the axial flow patterns of multi-periodic flows at $\mathrm{Dn}=220$ is potent than that at $\mathrm{Dn}=190$. At $\mathrm{Dn}=150$, the axial flow creates two high-velocity regions a single dumbbell at the upper wall of the duct (Fig. $4 \mathrm{~d}$ at $t=16.30$ ) while at $\mathrm{Dn}=190$ the axial flow produces two high-velocity regions with more than two dumbbells (Fig. 5d at $t=20.50$ ). This is occurred due to the centrifugal force of the duct. It is also described that due to the increase of dumbbells in axial flow, the number of secondary vortices is risen up. Two to five-vortex secondary velocity are generated at the outer, middle and lower wall of the duct. Thus, the secondary flow in multi-periodic solution also provides a basis for the mixing of fluids as well as heat transfer in the duct. It is well known that the vibrations of the particles in the ducts create instability within themselves. As a result, they are mixed well and when a fixed temperature is conducted throughout the duct wall and the particles, the particles certainly absorb the heat, and because of mixing themselves heat transfer is enhanced. However, in the present study, the streamlines in the temperature profiles demonstrate that the distribution of temperature has equally allocated from the outer heated wall, and due to the influence on curvature and heating-induced buoyancy force effect, secondary flows altered significantly and enhance the number of vortices in the duct. Besides, it is clear that the temperature contours are generated to the bottom wall of the duct but the heat transferred and mixed from the outer wall of the duct, as a consequence, asymmetric lines are caused which becomes relatively uneven than the isothermal flow. This is only constructed because of the connection between the centrifugal force and heating-induced buoyancy force which also affects the formation of secondary vortices. In this instance, it is said that the centrifugal force produces two different forces in the duct; one is, it drives the fluid motion from the inner wall to the outer wall of the duct and another is, it creates a pressure gradient region throughout the duct cross section. Here, the fluid motion happens opposite to the pressure gradient which is produced by the duct curvature and is imposed on the axial velocity to construct the secondary vortex. On the contrary, as the Dean number/pressure 


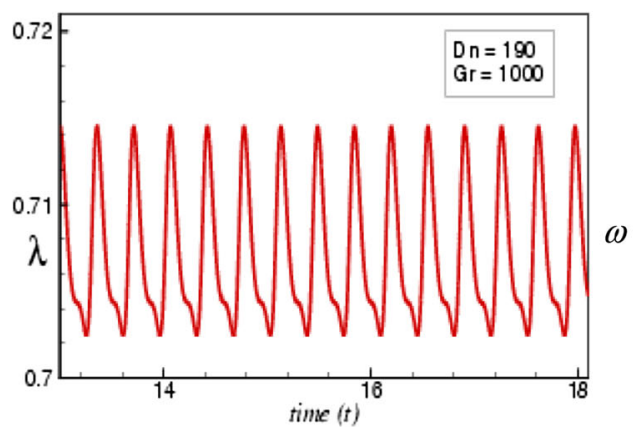

(a)

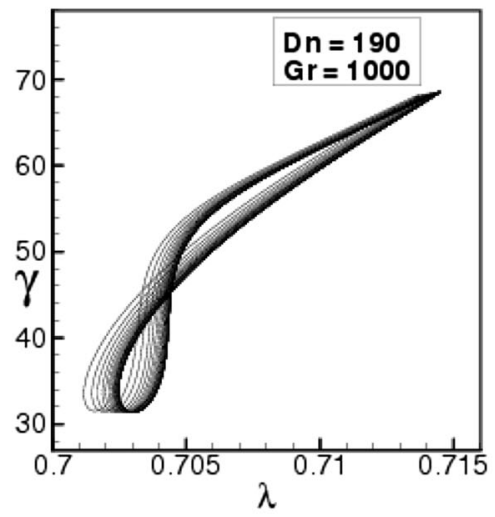

(b)

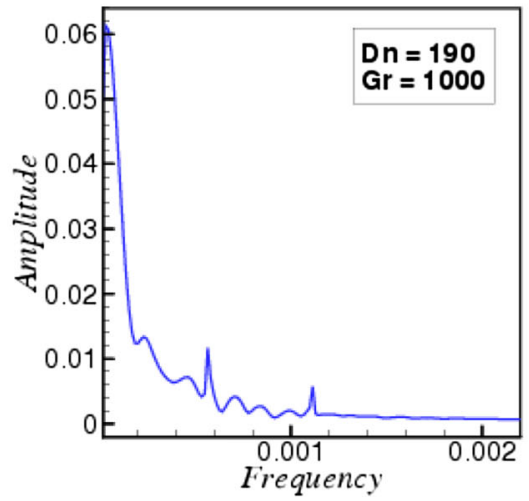

(c)
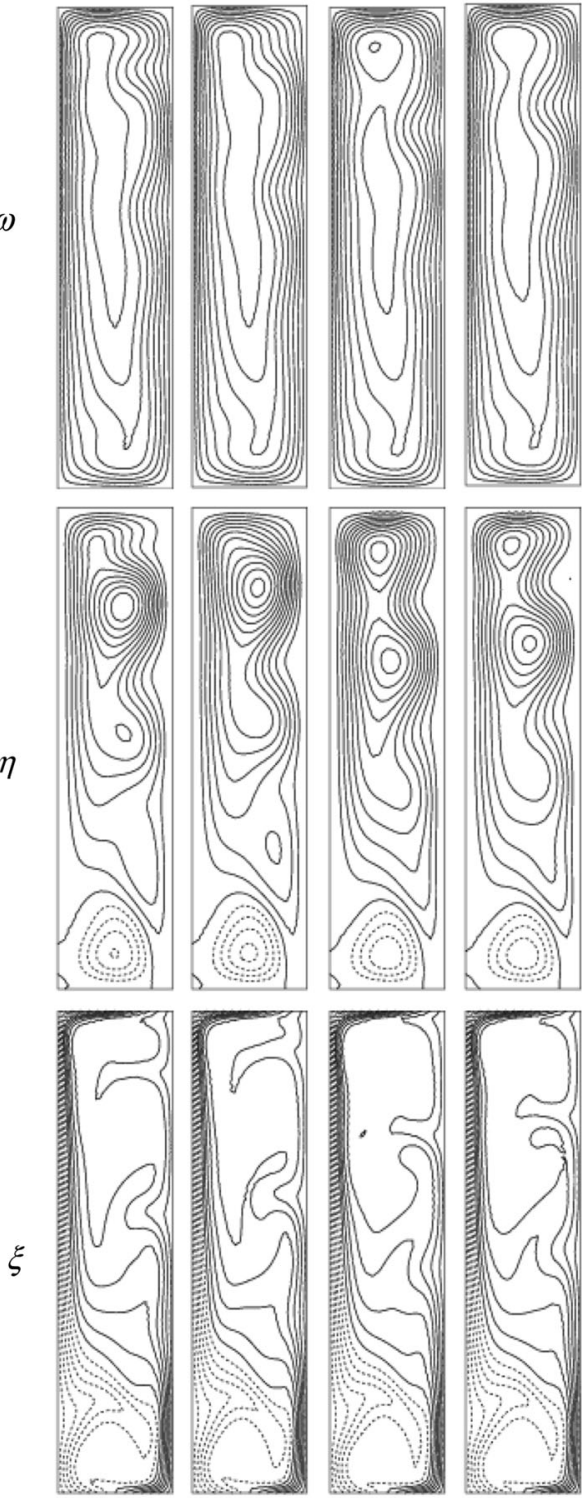

16.3

16.5

Fig. 4 a Unsteady solution, b phase space, c power spectrum, d axial (top) and secondary (middle) flow velocity and isotherms (bottom); for $\mathrm{Dn}=190$

gradient force is risen up, the flow velocities become stronger and the region of pressure becomes escalated. Alongside this, a lethargic flow region is formed because of the adverse pressure field and the viscosity effects reduce the fluid motion. On account of the increasing and decreasing of the flow, the pressure gradients become strong to the opposite flow direction 


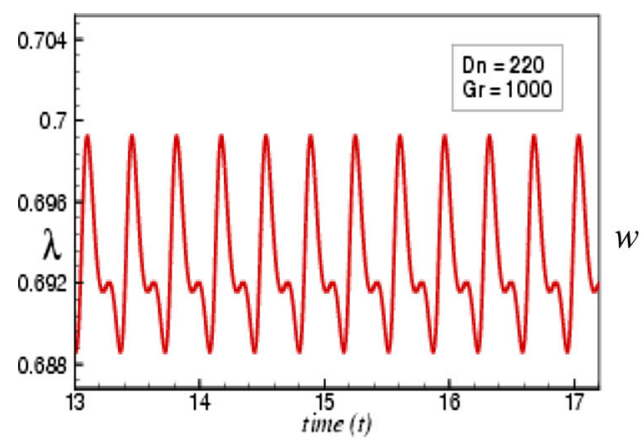

(a)

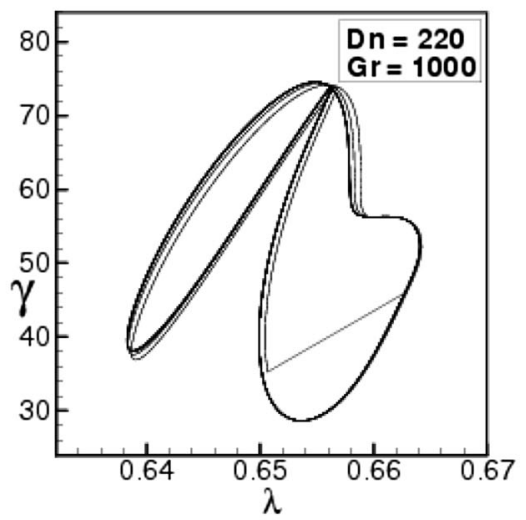

(b)

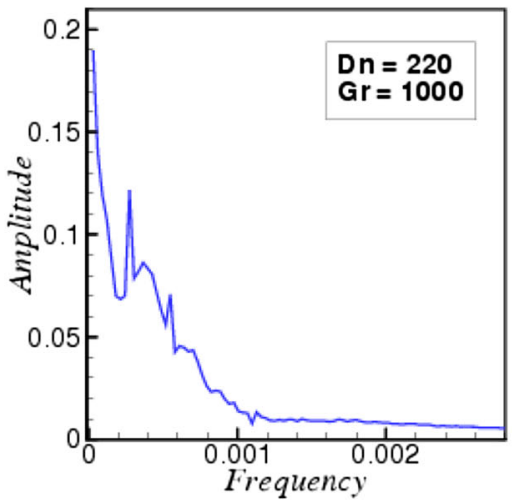

(c)
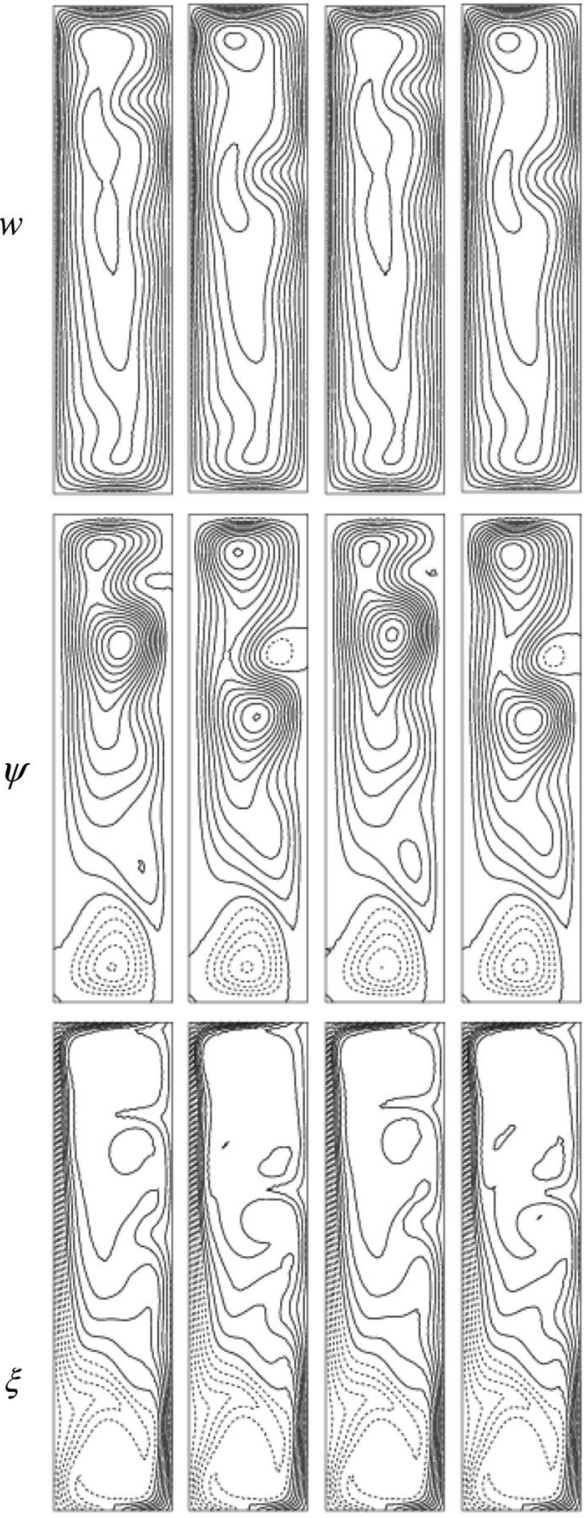

20.00

20.50

(d)

Fig. 5 a Unsteady solution, b phase space, c power spectrum, d axial (top) and secondary (middle) flow velocity and isotherms (bottom); for $\mathrm{Dn}=220$

under a certain critical value of Dn. As a result, new vortices of secondary flow appear at the outer wall of the duct. The newly two vortices are entitled as Dean vortex and the flow conditions are known as Dean's hydrodynamic instability where the previous two-vortex is called Ekman vortex. 


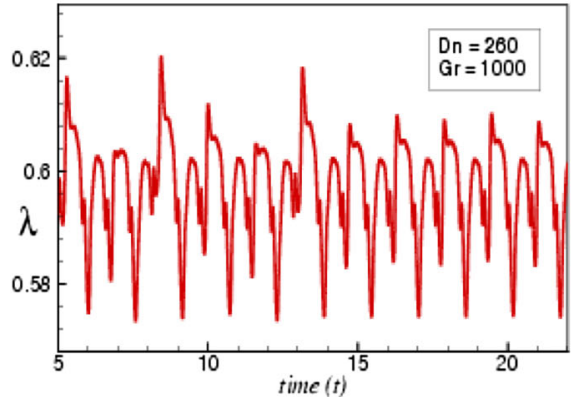

(a)

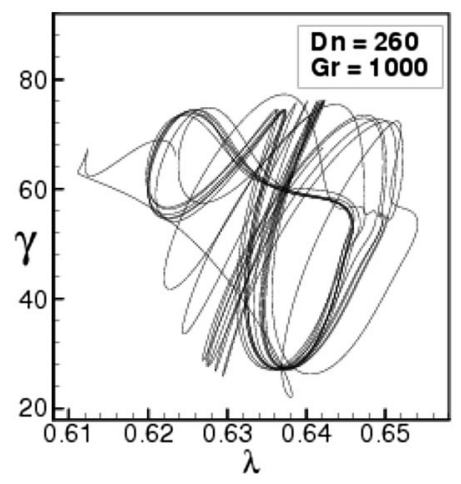

(b)

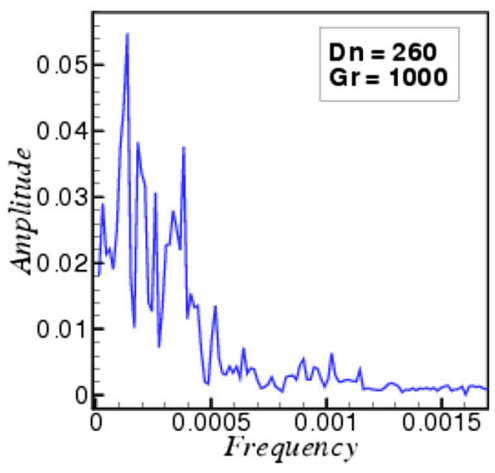

(c)
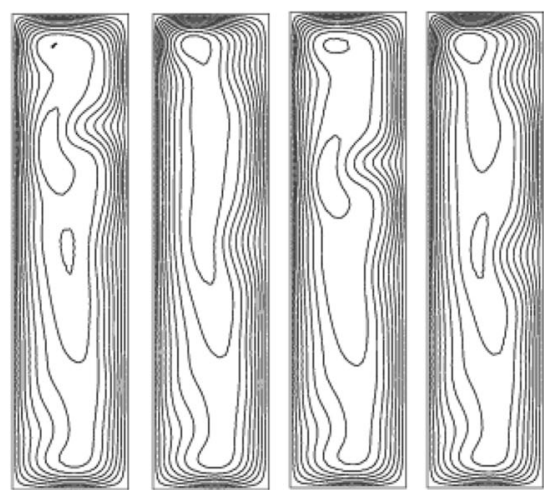

$\eta$
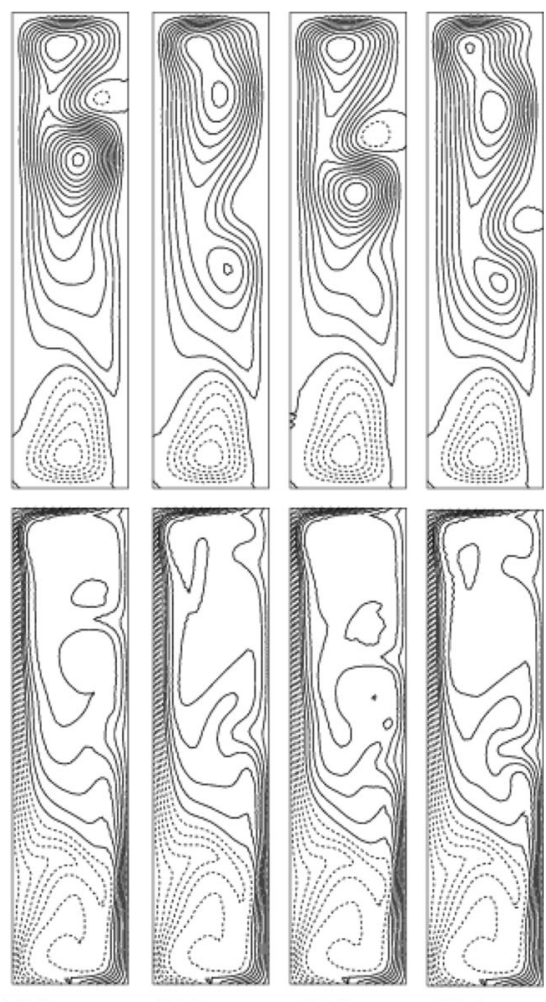

17.0

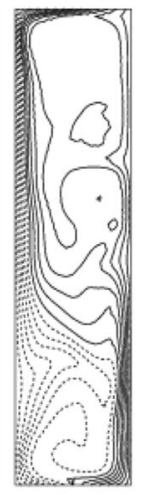

17.5

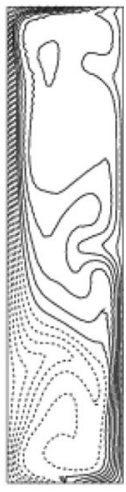

18.0

(d)

Fig. 6 a Unsteady solution, b phase space, c power spectrum, d axial (top) and secondary (middle) flow velocity and isotherms (bottom); for $\mathrm{Dn}=260$

Now, we analyze the time-dependent solution from $\mathrm{Dn}=230$ to $\mathrm{Dn}=260$ and it is detected that the unsteady flow behavior shows chaotic oscillation as shown in Fig. 6a for Dn $=260$. To have a clear insight into multi-periodic behavior, phase space and power spectrum are enumerated as disclosed in Fig. 6b, c, respectively. It is noted that the vibration as well as path lines and line spectrums of the particles are enhanced for increasing Dn. The phase space 
reports that the path lines of the stream flow start to move arbitrarily and show an irregular movement of the particles. But the density of the path lines is not enough what is considered to the chaotic oscillation. On the other side, the power spectrum discusses that the line spectrums oscillate subsequently and this oscillation is reduced when the line spectrum crosses 0.0002 in the frequency line. So this type of chaotic oscillation is termed as transitional chaos (details of transitional chaos are described by Mondal et al. [12]. Flow velocities such as axial and secondary flow and isotherms are sketched in Fig. 6d. It is obtained that high-velocity regions are produced in the axial flows, as a consequence, two- to five-vortex secondary flows are generated every side of the duct wall except the inner wall.

The transitional chaos is changed to multi-periodic again if Dn is raised up. The multiperiodic solutions occurred again at $\mathrm{Dn}=270$ as exhibited in Fig. 7a. The phase space and power spectrum for multi-periodic solutions are manipulated in Fig. 7b, c consecutively. The power spectrum in Fig. 7c shows that the line spectrum vibrates continuously but the line spectrums are weaker enough. The continuous vibrations happen due to the pressure gradient effect. But the phase space in Fig. 7b visualizes that the path lines completed two cycles in the lambda-gamma plane. Axial and secondary velocity and isotherms are displayed in Fig. 7d. Secondary vortices explain that due to the decrease of flow transition, the number of vortices reduces. From two- to four-vortex solution is found in the multi-periodic flow around the duct walls. A correlation has been established between the axial and the secondary velocity. At $t=12.70$, the axial flow pushes to the middle-outer wall of the duct and it creates two high-velocity regions with a dumbbell at the upper wall of the duct; and as a consequence, four-vortex secondary flow is obtained where the upper vortex is stronger than the lower one.

Now, time-dependent characteristics are explored for further Dn's. It is obtained that the chaotic oscillations start for prolonging Dn's after the multi-periodic oscillation. The chaotic characteristic begins at $\mathrm{Dn}=290$ and it is continued up to $\mathrm{Dn}=1000$. It is noticed that there is some dramatic change between these chaotic behaviors. However, Figs. 7a and 8a represent the chaotic flow for $\mathrm{Dn}=290$ and $\mathrm{Dn}=1000$, respectively. It is observed that the oscillations occurred in Figs. 7a and $8 \mathrm{a}$ is much stronger than that in Fig. 6a. So, the chaotic oscillation at $\mathrm{Dn}=290$ \& $\mathrm{Dn}=1000$ can be addressed as "strong chaos" (details of transitional chaos are described by Mondal et al. [12]). To fathom appropriately, phase space followed by power spectrums are evaluated further as depicted in Fig. 7b, c and in Fig. 8c, d consecutively. It is observed that the path line at $\mathrm{Dn}=2190$ moves willingly, and as a consequence it overlaps each other. The line spectrum for the same Dean number demonstrates that the beginning of frequency and the line spectrums oscillate strongly and the oscillation is weaker when it crosses 0.002 in the frequency line. On the other hand, phase space at $\mathrm{Dn}=1000$ elucidates that the path line oscillates more and creates a denser area than that at $\mathrm{Dn}=290$. Power spectrum for the same Dn interprets that the line spectrums oscillate frequently and the frequencies are stronger more than at $\mathrm{Dn}=290$. It is noticed that the total area of phase space and power spectrums are increased gradually for increasing the Dean number. So it can be easily said from not only phase space and power spectrum but also timehistory analysis that the chaotic flows are less stronger at low Dn's than the high Dn's. This is occurred due to different types of phenomena. The pressure of the fluid is higher due to the centrifugal force effect; besides that, the flow through the curved duct pushes to the outer wall while the temperature induces at the outer wall, therefore heating-induced buoyancy force is formed and the fluid is mixed properly which enhanced the flow vibration consequently. Moreover, we can also get a decision by depicting the flow velocity and the isotherms. Flow velocity including axial and secondary flow and isotherms are shown in Figs. $7 \mathrm{~d}$ and $8 \mathrm{~d}$ for $\mathrm{Dn}=290$ and $\mathrm{Dn}=1000$, respectively. It is seen that from two- to six-vortex secondary flow is produced for $\mathrm{Dn}=290$ and from two- to eight-vortex secondary flow is generated for 


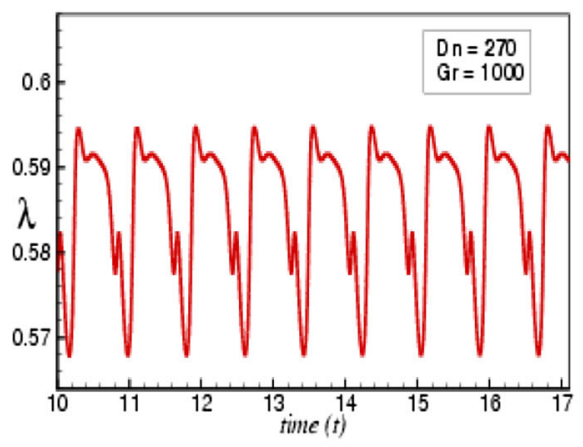

(a)

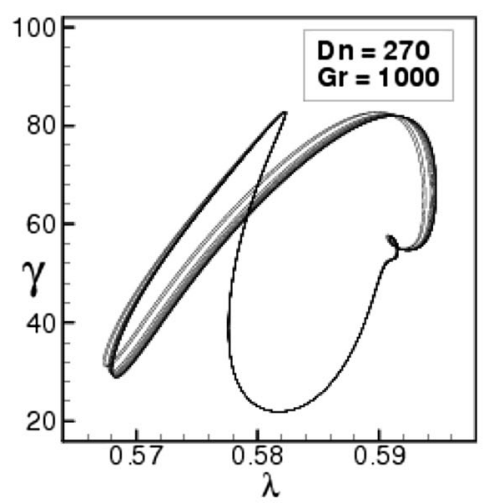

(b)

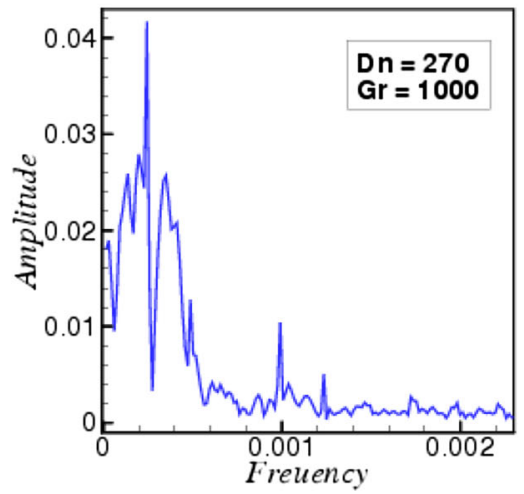

(c)
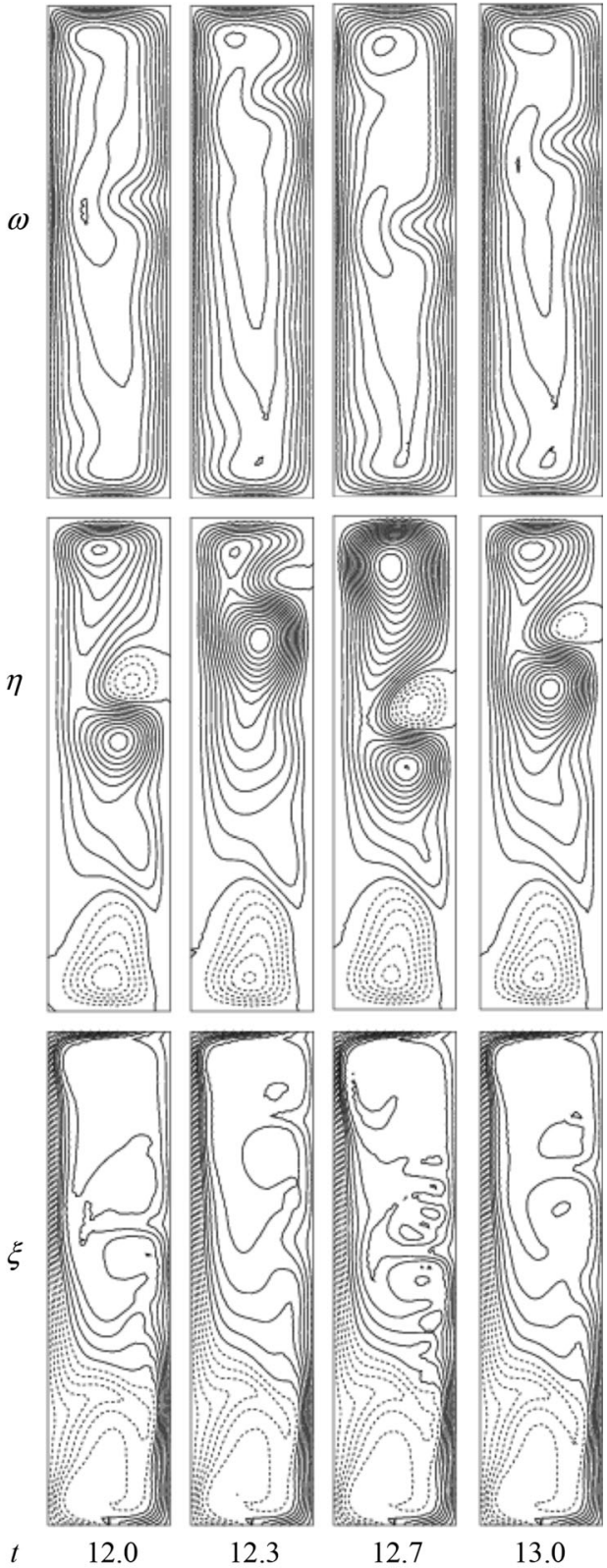

12.3

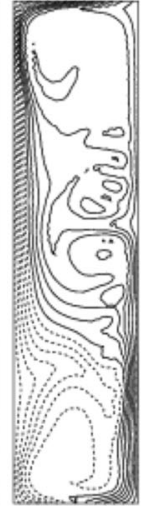

12.7

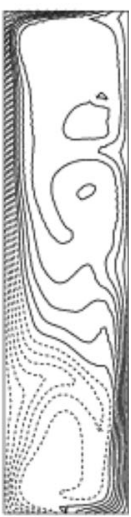

13.0

(d)

Fig. 7 a Unsteady solution, b phase space, c power spectrum, d axial (top) and secondary (middle) flow velocity and isotherms (bottom); for $\mathrm{Dn}=270$

$\mathrm{Dn}=1000$. In the chaotic flow at $\mathrm{Dn}=290$, a relationship is obtained among the axial and secondary flow. It is already noticed that the number of dumbbells in axial flow represents how strong the flow velocity is. single-, two- and three-dumbbells are created at the chaotic 

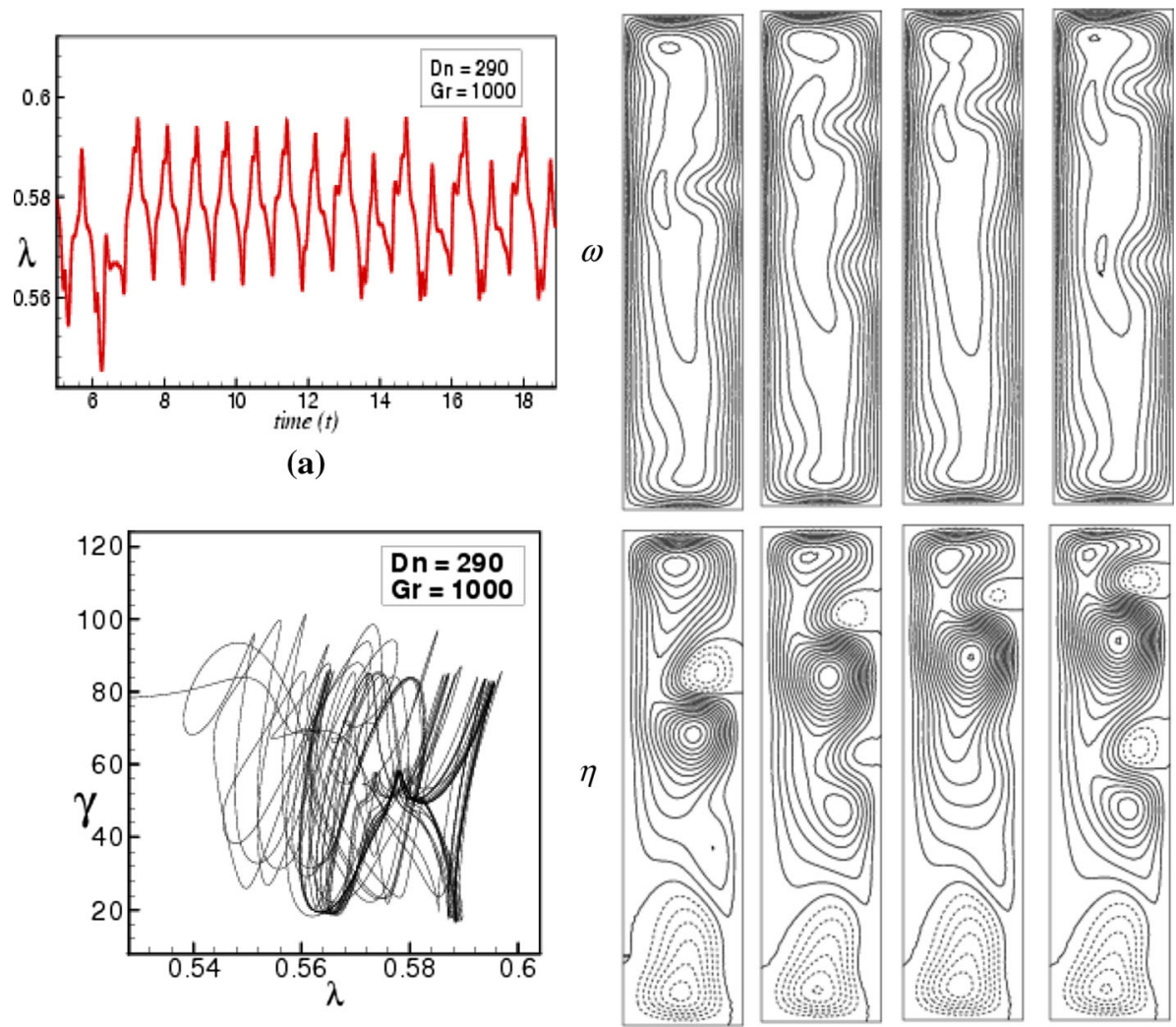

(b)

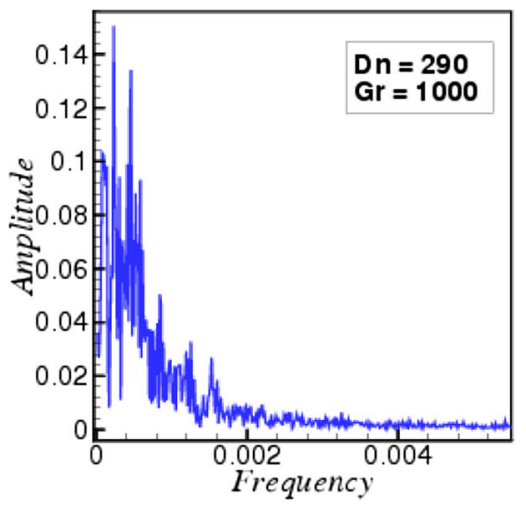

(c)
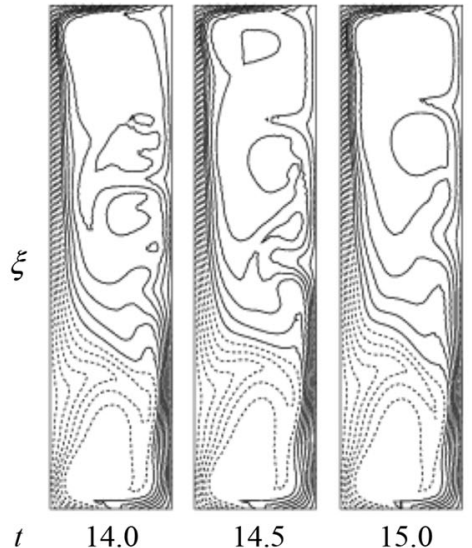

15.0

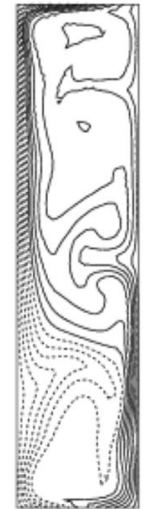

15.5

(d)

Fig. 8 a Unsteady solution, b phase space, c power spectrum, d axial (top) and secondary (middle) flow velocity and isotherms (bottom); for $\mathrm{Dn}=290$

flow. It is seen that when two dumbbells are generated at the axial flow, four-vortex is formed in the secondary flow, in the same way, when three dumbbells are found the secondary flow shows six-vortex. In a word, for increasing a single dumbbell in axial flow, an additional pair 
of vortex is generated in secondary flow. It is moreover analyzed that the dumbbells in the axial velocity at $t=15.50(\mathrm{Dn}=290)$ are created almost upper side of the duct wall, and consequently the newly secondary vortices are originated at the upper section of the duct. But it is noted that at $\mathrm{Dn}=1000$, the secondary vortex almost follows the rules in such a way as the number of vortex increased. At $t=12.50$, a dumbbell is produced at the lower-inner wall of the duct; therefore, a new vortex is created at the lower-inner wall of the duct. However, it is certain that the flow velocities are stronger at $\mathrm{Dn}=1000$ than that at $\mathrm{Dn}=290$ which can be easily proved by analyzing the number of dumbbells and the vortices. The isotherms show that the fluid is properly mixed and creates more density, which assures that buoyancy forces as well as overall heat in the duct are significantly transferred to the particle to particle (Fig. 9).

\subsection{Vortex structure}

Vortex structure explores the fluid mixing and the overall heat transfer in the fluid through the duct. In this section, a total vortex structure over a wide range of Dn $(100 \leq \mathrm{Dn} \leq 1000)$ is presented in Fig. 10 in Dn vs. Number of vortex plane. Here, single- to eight-vortex secondary flows are designated by circle, triangle, right triangle, square, left triangle, gradient, diamond and star, respectively. It is obtained that only single- and two-vortex solutions have generated for low Dn and steady-state solution. When the disturbance in the fluid particle occurs, the number of vortices increases as shown in Fig. 10 for $\mathrm{Dn}=200$. Three different types of asymmetric vortices are found at $\mathrm{Dn}=200$. The number of vortices is more increased in the multi-periodic and chaotic oscillation than the steady-state and periodic oscillation. It is seen that from two- to six-vortex solutions are produced for $300 \leq \mathrm{Dn} \leq 400$, two- to seven-vortex for $500 \leq \mathrm{Dn} \leq 800$ and above seven-vortex solutions are found for $900 \leq \mathrm{Dn} \leq 1000$, respectively. The consecutiveness of the increasing the number of vortices has occurred due to different types of forces including the centrifugal force which is induced by the curvature of the duct, pressure gradient force and the heating-induced buoyancy force and it is important to note that a relationship is built up among these three types of forces. In the steady-state solutions, no vibration occurs, so the particle moves without any collisions between them. When steady-state solution is changed to periodic oscillation, the number of vortices increases at $\mathrm{Dn}=200$ on account of its oscillation. Besides, colliding with the particles, the fluids are mixed which enhances the heat transfer overall in the fluid particles through the duct. In the multi-periodic $(\mathrm{Dn}=220)$ and transitional chaotic $(\mathrm{Dn}=230)$ oscillation, the number of vortices does not rise up but the secondary vortex is stronger than the steady-state solution certainly. So it can be said that as the pressure gradient of the fluid particle is not so high, the fluid particles do not composite with each other as expected. For increasing the pressure gradient parameter, the number of vortex is increased again in the chaotic oscillation and it is finally showed eight-vortex at $\mathrm{Dn}=1000$. So from $\mathrm{Dn}=500$, three types of forces act together, i.e., pressure gradient forces create the pressure between the fluid particles; colliding with fluid particles produced fluid mixing, besides it enhances the overall heat particularly vibration between the fluid particle created heating-induced buoyancy force; and finally, with this two types of forces, centrifugal force helps to increase the number of vortices. It is also noted that increasing the Dean number not only intensifies the number of vortices but also describes how much stronger the number of vortices and the chaos is. 


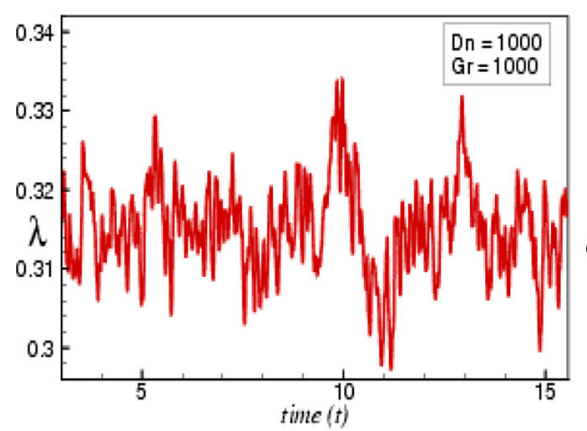

(a)

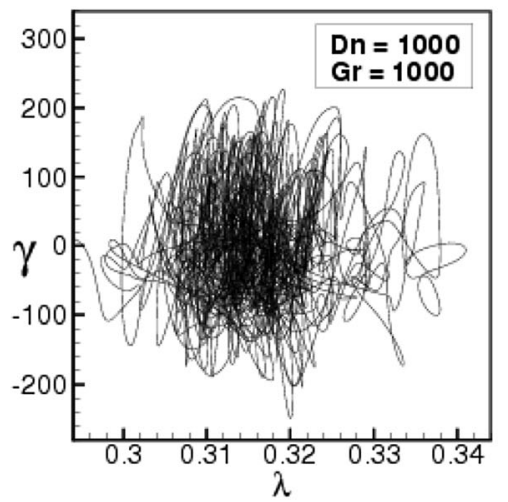

(b)

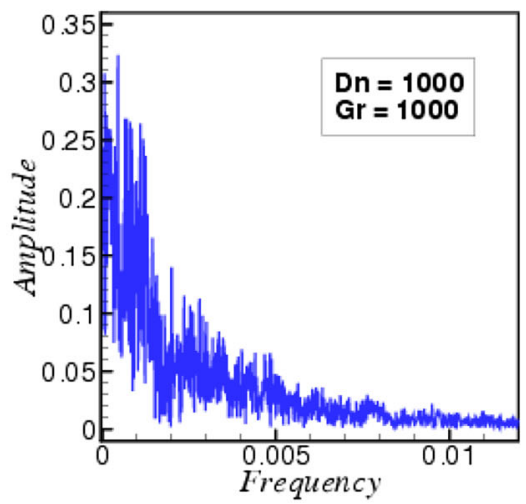

(c)

$\eta$
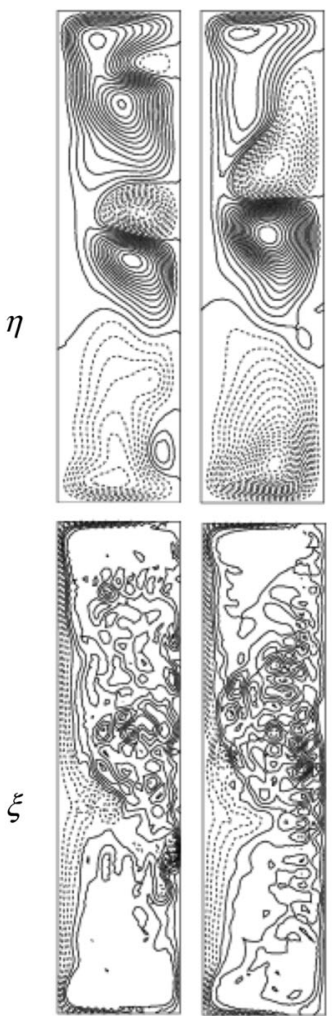

11.5
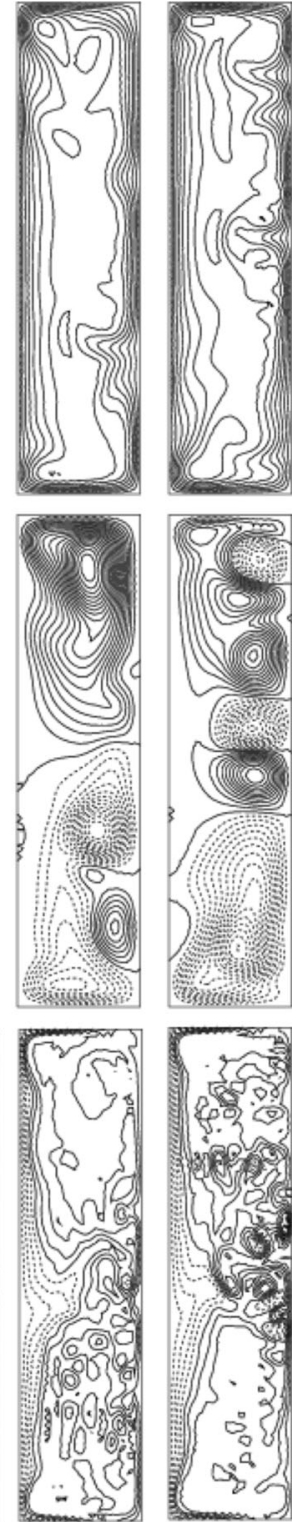

12.0

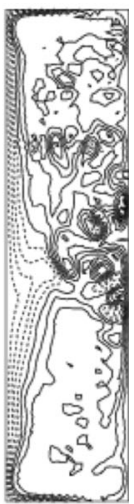

12.5

(d)

Fig. 9 a Unsteady solution, b phase space, c power spectrum, d axial (top) and secondary (middle) flow velocity and isotherms (bottom); for $\mathrm{Dn}=1000$

\subsection{Heat transfer (HT) and temperature gradients (TG)}

Heat transfer and the temperature gradients interpret the amount of heat transfer in the steady and unsteady solution throughout the duct as well as fluid particles. In this section, heat 
Fig. 10 Vortex structure of unsteady solutions for $100 \leq \mathrm{Dn} \leq 1000$

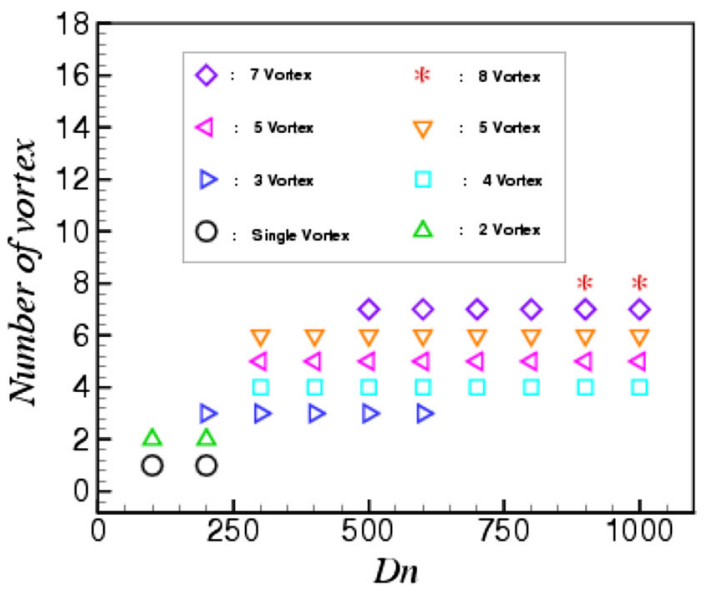

transfer is elucidated in Fig. 11a. From Fig. 11a, it is seen that two solid lines and circle and cross for $100 \leq \mathrm{Dn} \leq 1000$ are used to narrate the heat transfer. The solid lines are obtained from the steady solution branch which covers throughout the whole range of Dn. In the heat transfer figure, black and red solid lines represent the heat transfer for cooled and heated sidewall, respectively. On the other hand, the symbols are found from calculating the average of unsteady flow behavior for a specific Dn. The circles and cross address the average of timeevolution of the unsteady solution for cooled and heated side-wall consecutively. However, heat transfer in the rectangular curved duct demonstrates that the heat is transferred less from the cooled side-wall than the heated side-wall. It is also observed that the heat transfer in flow transition is enhanced significantly in both cooled and heated side walls as shown by the symbols. Moreover, the heat is shifted less for steady-state flow than the periodic, multiperiodic and chaotic oscillation. On the other hand, due to the heating-induced buoyancy force and the centrifugal force, the heat transmission in both steady and unsteady solution is raised more. So, it is apparent that the flow isolation at the heated side-wall consent the necessary heat transfer at the high flow rate (Dn) under fixed heat flux condition.

To have a clear insight into the heat transfer, temperature gradients are further calculated as an index of heat transfer as plotted in Fig. 11b, c for cooled and heated sidewalls, respectively. Temperature gradients for cooled and heated sidewalls demonstrate that the amount of heat is symmetric with respect to $y=0$. Temperature gradient for cooled side-wall describes that $\frac{\mathrm{d} T}{\mathrm{~d} x}$ enhances for raising Dn. It is also obtained that the heat is increased in a parallel way when Dn is passed over 100 and the heat plotted U-shape around $y=0$. On the contrary, temperature gradients for heated sidewall explore that the heat is increased more for raising Dn, but when the heat is crossed over -0.08 in the y-axis heat transfer becomes less and again heat transfer is increased when it passes -0.05 in y-axis and finally it climbs at the highest position when $y=0$. The same phenomenon is occurred on the right side of $y=0$. It is also noted for the heated side-wall that from $y=-0.5$ to $y=0.5, \frac{\mathrm{d} T}{\mathrm{~d} x}$ creates the opposite of U-shape. Mainly the increase of heat-flux at the central region occurs for two reasons; one is the advection of the duct and the other is the centrifugal force. More specifically, due to the aspect ratio, curvature, pressure gradient and advection, the secondary flow becomes stronger and because of this fluid is mixed up substantially and thereby enhances heat transfer in the fluid. Consequently, it is suggested that secondary flow creates an inward direction which is also known as the reverse flow of the outward secondary flow in the central region. 


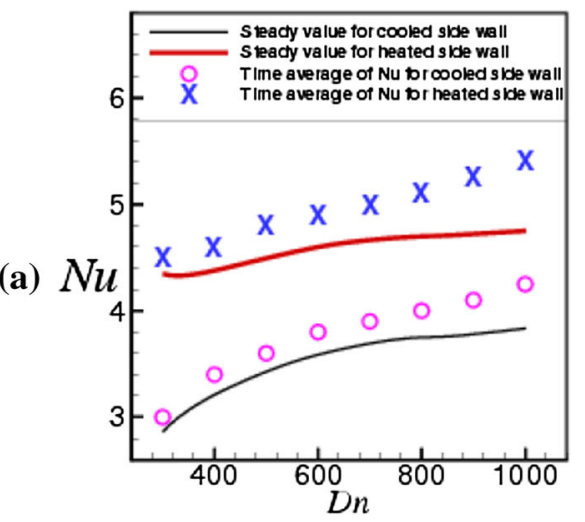

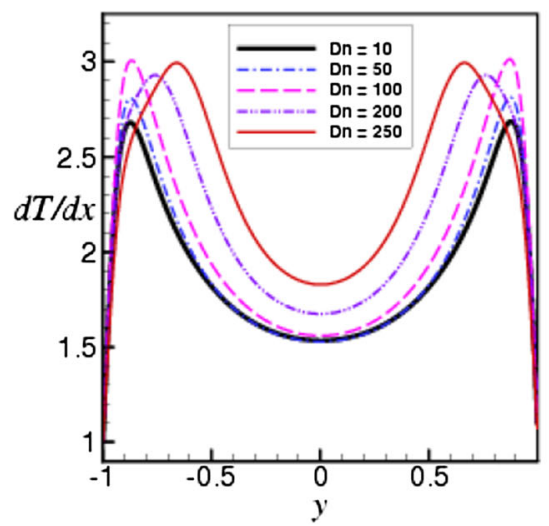

(b)

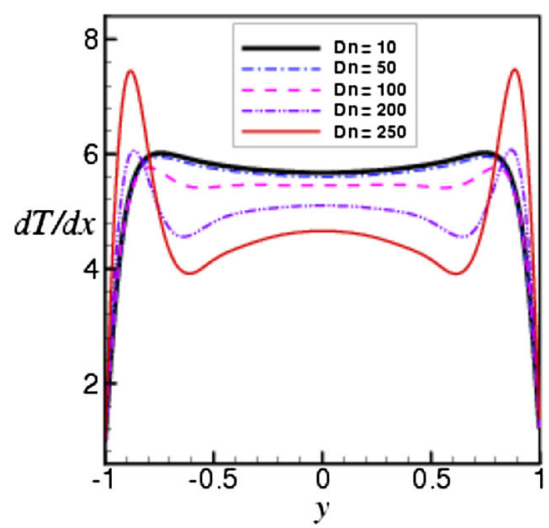

(c)

Fig. 11 a Heat transfer, b temperature gradients for cooled side-wall, c temperature gradient for heated sidewall; for several Dean numbers

\subsection{Experimental validation}

Now, the present numerical calculations are validated with the experimental outcome where the experimental outcomes are found by Chandratilleke et al. [3-5]. It should be noted that Chandratilleke et al. [3-5] first reveal the experimental outcome of the secondary flow for different aspect ratios (aspect ratio 2, 4 and 8) and non-isothermal cases for non-rotating curved duct. To visualize the outcome of the flow patterns, they have driven the flow visualization technique at $180^{\circ}$ inlet. Here, we compare our computational outcome with the experimental result. Figure 12 reveals the numerical vs. experimental results while right figures that is Fig. 12a-d exhibit numerical results, and the left figures show the experimental outcomes. On the other hand, Fig. 12a, b compares the numerical as well as experimental results for aspect ratio 2. It is noted that Fig. 12c, d illustrates not only the secondary flow but also the isotherms. However, it is observed that numerical outcomes of secondary flows and isotherm for different aspect ratios are quite identical with the experimental data. 

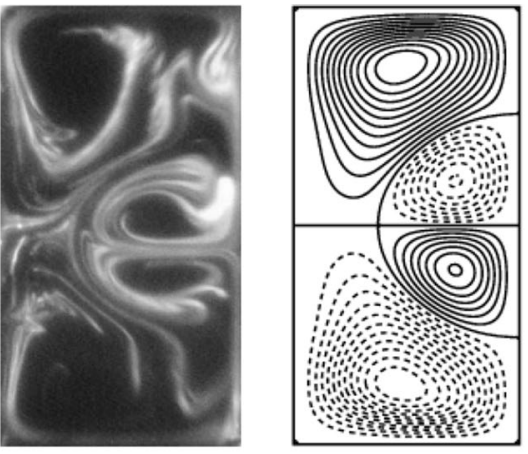

$K=262$
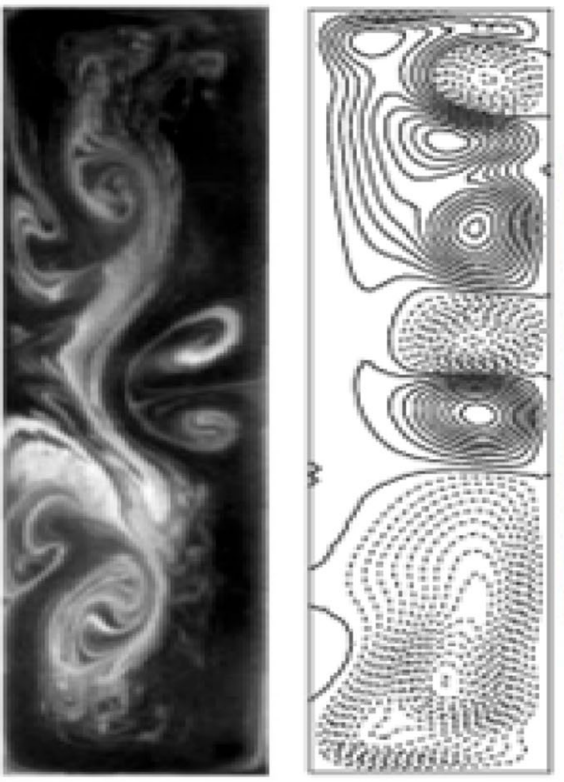

(c)
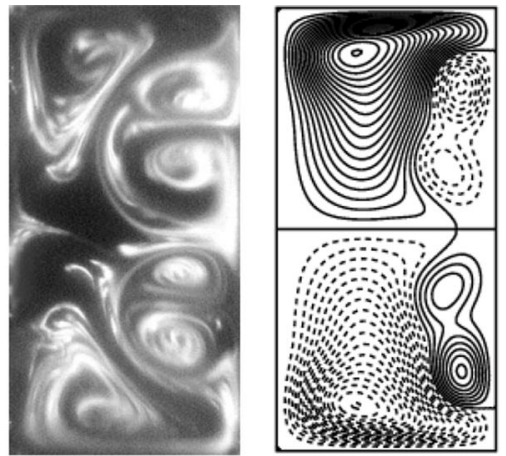

$K=321$

(b) $D n=317$
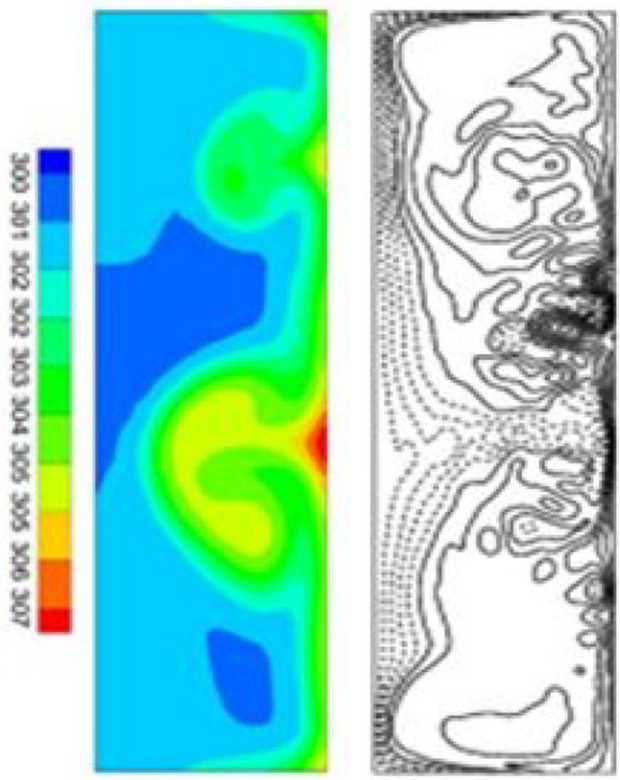

$K=375$

$D n=378$

Fig. 12 Comparison of experimental findings (left) with numerical result (right). a, b for aspect ratio 2, c, d for aspect ratio 4

\section{Discussions}

Flow transition and HT in a CRD is commonly used in industrial field including solar panels, metallic industry, polymer industry and air conditioning equipment. It is important that the engineers mainly look into optimizing the equipment costs as well as convenience of the usage of rectangular ducts before their experiments. For the applications in mechanical and chemical engineering, a curved rectangular-shaped duct is the best way for the researchers to optimize costs and convenience; for example, to make an air-condition, the most efficient way in terms of conveying air is to use the rectangular-shaped duct. On the other hand, a duct of large aspect ratio is the most proficient in the use of the material and experiences great 
pressure losses. Besides, the rectangular duct is ideal in trading off added duct cost of material and fan energy for headroom savings. Therefore, keeping these widespread applications of curved rectangular-shaped channels in mind, in this part, a brief discussion is apprehended along with the novelty of the present study. Watanabe and Yanase [59] numerically deployed the bifurcation, stability and flow-state features through a CSD. Though their study explored three-dimensional flow features for large Dn's but their study carried some limitations to present other phenomena like transitional behavior, influences of Dn and curvature on vortexpattern etc. Very recently, Nowruzi et al. [60] adopted CFD-based software using energy gradient method to visualize the hydrodynamic unsteadiness in the $\mathrm{CD}$ for various Ar where aspects of unsteady behavior with the influences of Ar on fluid transition were absent. But it is important that there is a deep connection between the unsteady behavior and hydrodynamicinstability of the flow. Hasan et al. [57] conducted spectral method to illustrate the relation between bifurcation structure, linear stability and flow transitions properly for CSD flow. Secondary flow (SF) mechanisms in bifurcations and unsteady behavior have been discussed by Liu and Wang [25]. They have considered showing this mechanism for large Ar but their study was unable to report effects of SF in HT. Even if the change of axial flow behavior with changing Dn and SF were absent in their study. Li et al. [44] investigated SF variation for $\delta=0.4,1$ and 2.3 In their study, they first discussed 3D contour and explored pointby-point variation in Dean flow for changing Ar. A suitable comparison is observed with their numerical and experimental data but HT impacts on the Dean flow was a lacking in their study. Norouzi and Biglari [33] computed the Dean flow analytically for a CRD by adopting the perturbation approach. A correlation of the HT and pressure drop for three different ducts containing coiled, circular and curved duct has been analyzed by Ghobadi and Muzychka [61] for large interval of Dn. Bouzit et al. [62] attempted to interpret the thermal-buoyancy effect in the curved duct flow and showed the isotherms and velocity contours separately by adopting the ANSYS-CFS software. Adopting computational fluid dynamics tool, Sasmito et al. [63] obtained different flow unsteadiness in nanofluid flow through coiled square tubes where they drew their velocity contours by using vector plots. Five different types of contours such as isotherms, masslines, heat-lines, streamlines and isosolutes for different Rayleigh numbers were presented by Kushawaha et al. [36]. Arpino et al. [64] elucidated the transient behavior of temperature contour for different aspect ratios. Very recently, Yadegari et al. [65] comprehensively studied the centrifugal force, turning angle and curvature ratio of the curved diffusers with rectangular cross section. Though they threedimensionally expressed all the phenomena of the channel but their study had a shortcoming to represent the relationship between axial, secondary and temperature contours as well as transient behavior of the flow in the channel. A detailed discussion on curved duct flow and HT was elucidated by Chandratilleke et al. [3-5] while they considered both cylindrical and elliptical coordinates in their exploration. Furthermore, they reported the thermal behavior and effect of secondary flow both numerically and experimentally. But their investigation was limited to low Dn. Though recently transitional behavior for two different pressure gradient parameters with buoyancy effects has been presented by Mondal et al. [26] for various Ar, but the impacts of periodic, multi- periodic and even chaotic behavior in the flow velocity were not explained elaborately in their study. In a word, a compact investigation on flow transition with the physics of fluids; the correlation between axial, SF velocity and isotherms are not demonstrated together in the above exploration. Therefore, our current study aimed to meet up this lacking information because till now no complete investigation is available for transient solutions with flow transition for a stationary CRD taking large Ar effects on vortex-pattern into account which has been addressed in the existing study very meticulously. In addition, hydrodynamic-unsteadiness and production of longitudinal vortex have been discussed in 
the ongoing study which gives a strong conception about the convective HT through a CRD via flow state oscillations. The ongoing exploration furthermore calculates the overall HT including TG and fluid mixing in a large-shaped rectangular duct in detail.

\section{Conclusion}

A mathematical analysis for transient fluid flow with HT has been presented through a CRD of strong curvature with large Ar over a wide range of the Dean number $(0<\mathrm{Dn} \leq 1000)$. The outer side-wall is heated and the other three walls are at room temperature. The below stated points are major outcomes of this study.

- The transient flow develops in the sequence "steady-state $\rightarrow$ periodic $\rightarrow$ multi-periodic $\rightarrow$ chaotic $\rightarrow$ multi-periodic $\rightarrow$ chaotic," if Dn is increased. The oscillating behavior shows that it gradually increases with the increase of Dn. The phase spaces for the periodic and multi-periodic flow show the single and multiple orbits, respectively, whereas chaotic oscillations create episodic path lines. The line spectrum reveals that the frequencies are fluctuated remarkably for chaotic oscillation than periodic and multi-periodic oscillation.

- Flow velocity and isotherms show several behaviors of the flow features. It is revealed that the flow velocity and the isotherms are significantly influenced by the duct curvature and the aspect ratio of the duct.

- The study shows that only single- and two-vortex secondary flow is created at no oscillation state flow, two- to six-vortex for periodic oscillation while two- to eight-vortex for irregular oscillation. A dominant link is established among the axial flow, secondary flow and isotherms. The isotherms depict that the fluid particles transfer heat from the heated wall and the normal temperature fluid, and they are mixed significantly with each other and consequently heating-induced buoyancy force is enhanced. The flow velocity is affected causing the centrifugal force greatly.

- The overall heat transfer in the periodic, multi-periodic and chaotic oscillation is expressively more than the steady-state solution which gives a firm framework that the fluid is mixed at a great deal for the regular and irregular oscillation. When the two forces, centrifugal and buoyancy, are accumulated, the flow becomes stronger creating multi-vortex solution and thereafter promotes heat transfer in the fluid.

Funding Open access funding provided by Università degli Studi di Parma within the CRUI-CARE Agreement.

Open Access This article is licensed under a Creative Commons Attribution 4.0 International License, which permits use, sharing, adaptation, distribution and reproduction in any medium or format, as long as you give appropriate credit to the original author(s) and the source, provide a link to the Creative Commons licence, and indicate if changes were made. The images or other third party material in this article are included in the article's Creative Commons licence, unless indicated otherwise in a credit line to the material. If material is not included in the article's Creative Commons licence and your intended use is not permitted by statutory regulation or exceeds the permitted use, you will need to obtain permission directly from the copyright holder. To view a copy of this licence, visit http://creativecommons.org/licenses/by/4.0/.

\section{References}

1. W.R. Dean, Note on the motion of fluid in a curved pipe. Lond. Edinb. Dublin Philos. Mag. J. Sci. 4, 208-223 (1927). https://doi.org/10.1080/14786440708564324

2. R.N. Mondal, Isothermal and Non-Isothermal Flows through Curved Ducts with Square and Rectangular Cross Section (Department of Mechanical and Systems Engineering. Okayama University, Japan, 2006). 
3. T.T. Chandratilleke, N. Nadim, R. Narayanaswamy, Vortex structure-based analysis of laminar flow behaviour and thermal characteristics in curved ducts. Int. J. Therm. Sci. 59, 75-86 (2012). https:// doi.org/10.1016/j.ijthermalsci.2012.04.014

4. T.T. Chandratilleke, N. Nadim, R. Narayanaswamy, Analysis of secondary flow instability and forced convection in fluid flow through rectangular and elliptical curved ducts. Heat Transf. Eng. 34, 1237-1248 (2013). https://doi.org/10.1080/01457632.2013.777249

5. T.T. Chandratilleke, Nursubyakto, Numerical prediction of secondary flow and convective heat transfer in externally heated curved rectangular ducts. Int. J. Therm. Sci. 42, 187-198 (2003). https://doi.org/10. 1016/S1290-0729(02)00018-2

6. M.M. Ahmadpour, M.A. Akhavan-Behabadi, Experimental investigation of heat transfer during flow condensation of HC-R600a based nano-refrigerant inside a horizontal U-shaped tube. Int. J. Therm. Sci. 146, 106110 (2019). https://doi.org/10.1016/j.ijthermalsci.2019.106110

7. N.H. Abu-Hamdeh, R.A.R. Bantan, I. Tlili, Analysis of the thermal and hydraulic performance of the sector-by-sector helically coiled tube heat exchangers as a new type of heat exchangers. Int. J. Therm. Sci. 150, 106229 (2020). https://doi.org/10.1016/j.ijthermalsci.2019.106229

8. S. Pourhedayat, S.M. Pesteei, H.E. Ghalinghie, M. Hashemian, M.A. Ashraf, Thermal-exergetic behavior of triangular vortex generators through the cylindrical tubes. Int. J. Heat Mass Transf. 151, 119406 (2020). https://doi.org/10.1016/j.ijheatmasstransfer.2020.119406

9. J.C. Umavathi, O.A. Bég, Effects of thermophysical properties on heat transfer at the interface of two immisicible fluids in a vertical duct: numerical study. Int. J. Heat Mass Transf. 154, 119613 (2020). https:// doi.org/10.1016/j.ijheatmasstransfer.2020.119613

10. S. Yanase, R.N. Mondal, Y. Kaga, Numerical study of non-isothermal flow with convective heat transfer in a curved rectangular duct. Int. J. Therm. Sci. 44, 1047-1060 (2005). https://doi.org/10.1016/j.ijthermalsci. 2005.03.013

11. S. Yanase, R. Nath Mondal, Y. Kaga, K. Yamamoto, Transition from steady to chaotic states of isothermal and non-isothermal flows through a curved rectangular duct. J. Phys. Soc. Jpn. 74, 345-358 (2005). https:// doi.org/10.1143/JPSJ.74.345

12. R.N. Mondal, Y. Kaga, T. Hyakutake, S. Yanase, Bifurcation diagram for two-dimensional steady flow and unsteady solutions in a curved square duct. Fluid Dyn. Res. 39, 413-446 (2007). https://doi.org/10.1 016/j.fluiddyn.2006.10.001

13. M.S. Hasan, M.M. Islam, S.C. Ray, R.N. Mondal, Bifurcation structure and unsteady solutions through a curved square duct with bottom wall heating and cooling from the ceiling. AIP Conf. Proc. 2121, 050003 (2019). https://doi.org/10.1063/1.5115890

14. S. Tanweer, A. Dewan, S. Sanghi, Influence of three-dimensional wake transition on heat transfer from a square cylinder near a moving wall. Int. J. Heat Mass Transf. 148, 118986 (2020). https://doi.org/10.101 6/j.ijheatmasstransfer.2019.118986

15. A. Hashemi, P.F. Fischer, F. Loth, Direct numerical simulation of transitional flow in a finite length curved pipe. J. Turbul. 19, 664-682 (2018). https://doi.org/10.1080/14685248.2018.1497293

16. A. Alonso, I. Mercader, O. Batiste, Time-dependent patterns in quasivertical cylindrical binary convection. Phys. Rev. E 97, 023108 (2018). https://doi.org/10.1103/PhysRevE.97.023108

17. N. Kurtulmuş, H. Zontul, B. Sahin, Heat transfer and flow characteristics in a sinusoidally curved converging-diverging channel. Int. J. Therm. Sci. 148, 106163 (2020). https://doi.org/10.1016/j. ijthermalsci.2019.106163

18. M.Z. Islam, R.N. Mondal, M.M. Rashidi, Dean-Taylor flow with convective heat transfer through a coiled duct. Comput. Fluids 149, 41-55 (2017). https://doi.org/10.1016/j.compfluid.2017.03.001

19. S.C. Ray, M.S. Hasan, R.N. Mondal, On the onset of hydrodynamic instability with convective heat transfer through a rotating curved rectangular duct. Math. Model. Eng. Probl. 7, 31-44 (2020). https:// doi.org/10.18280/mmep.070105

20. B. Zhou, J. Wang, Z. Yao, G. Zhang, X. Han, X. Wang, Vortex-induced vibration of a cylinder downstream of an elliptical cylinder with different aspect ratios. J. Mar. Sci. Technol. 25, 1044-1062 (2020). https:// doi.org/10.1007/s00773-019-00699-0

21. W. Zhang, Y. Wei, H.S. Dou, Z. Zhu, Transient behaviors of mixed convection in a square enclosure with an inner impulsively rotating circular cylinder. Int. Commun. Heat Mass Transf. 98, 143-154 (2018). https://doi.org/10.1016/j.icheatmasstransfer.2018.08.016

22. M.S. Hasan, R.N. Mondal, G. Lorenzini, Centrifugal instability with convective heat transfer through a tightly coiled square duct. Math. Model. Eng. Probl. 6, 397-408 (2019). https://doi.org/10.18280/mmep. 060311

23. M.S. Hasan, R.N. Mondal, G. Lorenzini, Numerical prediction of non-isothermal flow with convective heat transfer through a rotating curved square channel with bottom wall heating and cooling from the ceiling. Int. J. Heat Technol. 37, 710-726 (2019). https://doi.org/10.18280/ijht.370307 
24. S. Yanase, Y. Kaga, R. Daikai, Laminar flow through a curved rectangular duct over a wide range of aspect ratio. Fluid Dyn. Res. 31, 151-183 (2002). https://doi.org/10.1016/S0169-5983(02)00103-X

25. F. Liu, L. Wang, Analysis on multiplicity and stability of convective heat transfer in tightly curved rectangular ducts. Int. J. Heat Mass Transf. 52, 5849-5866 (2009). https://doi.org/10.1016/j.ijheatmasstransfer. 2009.07.019

26. R.N. Mondal, T. Watanabe, M.A. Hossain, S. Yanase, Vortex-structure and unsteady solutions with convective heat transfer through a curved duct. J. Thermophys. Heat Transf. 31, 243-254 (2017). https://doi. org/10.2514/1.T4913

27. J. Raja, F. Mebarek-Oudina, A.J. Chamkha, Magnetohydrodynamic flow of molybdenum disulfide nanofluid in a channel with shape effects. Multidiscip. Model. Mater. Struct. 15, 737-757 (2019). https:// doi.org/10.1108/MMMS-07-2018-0133

28. J.C. Umavathi, A.J. Chamkha, A. Mateen, A. Al-Mudhaf, Unsteady two-fluid flow and heat transfer in a horizontal channel. Heat Mass Transf. 42, 81 (2005). https://doi.org/10.1007/s00231-004-0565-X

29. S. Akram, Q. Afzal, Effects of thermal and concentration convection and induced magnetic field on peristaltic flow of Williamson nanofluid in inclined uniform channel. Eur. Phys. J. Plus 135, 857 (2020). https://doi.org/10.1140/epjp/s13360-020-00869-9

30. R.N. Mondal, M.S. Islam, K. Uddin, A. Hossain, Effects of aspect ratio on unsteady solutions through curved duct flow. Appl. Math. Mech. 34, 1107-1122 (2013). https://doi.org/10.1007/s10483-013-1731-8

31. R.N. Mondal, Y. Kaga, T. Hyakutake, S. Yanase, Effects of curvature and convective heat transfer in curved square duct flows. Trans. ASME J. Fluids Eng. 128, 1013-1022 (2006). https://doi.org/10.1115/ 1.2236131

32. M. Norouzi, M.H. Kayhani, M.R.H. Nobari, M.K. Demneh, Convective heat transfer of viscoelastic flow in a curved duct. World Acad. Sci. Eng. Technol. Int. J. Mech. Mechatron. Eng. 3, 921-927 (2009). https:// doi.org/10.5281/zenodo.1081071

33. M. Norouzi, N. Biglari, An analytical solution for Dean flow in curved ducts with rectangular cross section. Phys. Fluids 25, 053602 (2013). https://doi.org/10.1063/1.4803556

34. X.Y. Wu, S.D. Lai, K. Yamamoto, S. Yanase, Numerical analysis of the flow in a curved duct. Adv. Mater. Res. 706-708, 1450-1453 (2013)

35. H. Nowruzi, H. Ghassemi, S.S. Nourazar, Hydrodynamic stability study in a curved square duct by using the energy gradient method. J. Braz. Soc. Mech. Sci. Eng. 41, 288 (2019). https://doi.org/10.1007/s4043 $0-019-1790-z$

36. D. Kushawaha, S. Yadav, D.K. Singh, Thermo-solute natural convection with heat and mass lines in a uniformly heated and soluted rectangular enclosure for low Prandtl number fluids. Int. J. Therm. Sci. 148, 106160 (2020). https://doi.org/10.1016/j.ijthermalsci.2019.106160

37. M.S. Hasan, M.S. Islam, M.F. Badsha, R.N. Mondal, G. Lorenzini, Numerical investigation on the transition of fluid flow characteristics through a rotating curved duct. Int. J. Appl. Mech. Eng. 25, 45-63 (2020). https://doi.org/10.2478/ijame-2020-0034

38. M.N. Sultana, M.S. Hasan, R.N. Mondal, A numerical study of unsteady heat and fluid flow through a rotating curved channel with variable curvature. AIP Conf. Proc. 2121, 030009 (2019). https://doi.org/1 $0.1063 / 1.5115854$

39. R. Liu, H. Li, R. You, Z. Tao, Experimental investigation of turbulent flow in a two-pass channel with different U-shaped bends. AIP Adv. 10, 065311 (2020). https://doi.org/10.1063/5.0011444

40. L. Zhang, X. Yan, Y. Zhang, Y. Feng, Y. Li, H. Meng, J. Zhang, J. Wu, Heat transfer enhancement by streamlined winglet pair vortex generators for helical channel with rectangular cross section. Chem. Eng. Process. 147, 107788 (2020). https://doi.org/10.1016/j.cep.2019.107788

41. K.S. Bibin, J.S. Jayakumar, Thermal hydraulic characteristics of square ducts having porous material inserts near the duct wall or along the duct centre. Int. J. Heat Mass Transf. 148, 119079 (2020). https:// doi.org/10.1016/j.ijheatmasstransfer.2019.119079

42. L.Y. Zhang, Z. Lu, L.C. Wei, X. Yang, X.L. Yu, X.Z. Meng, L.W. Jin, Numerical investigation of flow characteristics and heat transfer performance in curve channel with periodical wave structure. Int. J. Heat Mass Transf. 140, 426-439 (2019). https://doi.org/10.1016/j.ijheatmasstransfer.2019.05.117

43. Y. Li, X. Wang, S. Yuan, S.K. Tan, Flow development in curved rectangular ducts with continuously varying curvature. Exp. Therm. Fluid Sci. 75, 1-15 (2016). https://doi.org/10.1016/j.expthermflusci.201 6.01 .012

44. Y. Li, X. Wang, B. Zhou, S. Yuan, S.K. Tan, Dean instability and secondary flow structure in curved rectangular ducts. Int. J. Heat Fluid Flow 68, 189-202 (2017). https://doi.org/10.1016/j.ijheatfluidflow.2 017.10.011

45. L. Riyi, W. Xiaoqian, X. Weidong, J. Xinfeng, J. Zhiying, Experimental and numerical study on forced convection heat transport in eccentric annular channels. Int. J. Therm. Sci. 136, 60-69 (2019). https://doi. org/10.1016/j.ijthermalsci.2018.10.003 
46. C. Zhang, Y. Niu, J. Xu, An anisotropic turbulence model for predicting heat transfer in atating channel. Int. J. Therm. Sci. 148, 106119 (2020). https://doi.org/10.1016/j.ijthermalsci.2019.106119

47. H. Benzenine, R. Saim, S. Abboudi, O. Imine, H.F. Oztop, N. Abu-Hamdeh, Numerical study of a threedimensional forced laminar flow in a channel equipped with a perforated baffle. Numer. Heat Transf. Part A Appl. 73, 881-894 (2018). https://doi.org/10.1080/10407782.2018.1486645

48. A.J. Chamkha, A.S. Dogonchi, D.D. Ganji, Magneto-hydrodynamic flow and heat transfer of a hybrid nanofluid in a rotating system among two surfaces in the presence of thermal radiation and Joule heating. AIP Adv. 9, 025103 (2019). https://doi.org/10.1063/1.5086247

49. A.S. Dogonchi, M.K. Nayak, N. Karimi, A.J. Chamkha, D.D. Ganji, Numerical simulation of hydrothermal features of $\mathrm{Cu}-\mathrm{H}_{2} \mathrm{O}$ nanofluid natural convection within a porous annulus considering diverse configurations of heater. J. Therm. Anal. Calorim. 141, 2109-2125 (2020). https://doi.org/10.1007/s10973020-09419-y

50. N.S. Shashikumar, S. Gireesha, B. Mahanthesh, B.C. Prasannakumara, A.J. Chamkha, Entropy generation analysis of magneto-nanoliquids embedded with aluminium and titanium alloy nanoparticles in microchannel with partial slips and convective conditions. Int. J. Numer. Methods Heat Fluid Flow 29, 3638-3658 (2019). https://doi.org/10.1108/HFF-06-2018-0301

51. N. Fallahnezhad, H.R. Nazif, Heat convection and hydrodynamic analysis of laminar developing nanofluid channel flow with variable properties under constant magnetic field and heat flux. Eur. Phys. J. Plus 134, 409 (2019). https://doi.org/10.1140/epjp/i2019-12787-6

52. F. Selimefendigil, H.F. Öztop, A.J. Chamkha, Role of magnetic field on forced convection of nanofluid in a branching channel. Int. J. Numer. Methods Heat Fluid Flow 30, 1755-1772 (2019). https://doi.org/1 0.1108/HFF-10-2018-0568

53. D. Baleanu, R. Sadat, M.R. Ali, The method of lines for solution of the carbon nanotubes engine oil nanofluid over an unsteady rotating disk. Eur. Phys. J. Plus 135, 788 (2020). https://doi.org/10.1140/epjp/ s13360-020-00763-4

54. Z. Yang, L. Ding, L. Zhang, L. Yang, H. He, Two degrees of freedom flow-induced vibration and heat transfer of an isothermal cylinder. Int. J. Heat Mass Transf. 154, 119766 (2020). https://doi.org/10.1016/ j.ijheatmasstransfer.2020.119766

55. G. Rajamohan, N. Ramesh, P. Kumar, Mixed convection and radiation studies on thermally developing laminar flow in a horizontal square channel with variable side heated wall. Int. J. Therm. Sci. 140, 298-307 (2020). https://doi.org/10.1016/j.ijthermalsci.2019.03.002

56. S.N. Dolon, M.S. Hasan, S.C. Ray, R.N. Mondal, Vortex-structure of secondary flows with effects of strong curvature on unsteady solutions through a curved rectangular duct of large aspect ratio. AIP Conf. Proc. 2121, 050004 (2019). https://doi.org/10.1063/1.5115891

57. M.S. Hasan, R.N. Mondal, G. Lorenzini, Physics of bifurcation of the flow and heat transfer through a curved duct with natural and forced convection. Chin. J. Phys. 67, 428-457 (2020). https://doi.org/10.10 16/j.cjph.2020.07.004

58. D. Gottlieb, S. A. Orazag, Numerical Analysis of Spectral Methods. Society of Industrial and Applied Mathematics. Siam, Philadelphia, USA (1977). https://doi.org/10.1137/1.9781611970425

59. T. Watanabe, S. Yanase, Bifurcation study of three-dimensional solutions of the curved square-duct flow. J. Phys. Soc. Jpn. 82, 074472 (2013). https://doi.org/10.7566/JPSJ.82.074402

60. H. Nowruzi, H. Ghassemi, S.S. Nourazar, Study of the effects of aspect ratio on hydrodynamic stability in curved rectangular ducts using energy gradient method. Eng. Sci. Technol. Int. J. 23, 334-344 (2020). https://doi.org/10.1016/j.jestch.2019.05.004

61. M. Ghobadi, Y.S. Muzychka, A review of heat transfer and pressure drop correlations for laminar flow in curved circular ducts. Heat Transf. Eng. 37, 815-839 (2015). https://doi.org/10.1080/01457632.2015. 1089735

62. M. Bouzit, O.D. Makinde, H. Laidoudi, M. Mokeddem, 3D Simulation of incompressible poiseuille flow through $180^{\circ}$ curved duct of square cross-section under effect of thermal buoyancy. Period. Polytech. Mech. Eng. 63, 257-269 (2019). https://doi.org/10.3311/PPme.12773

63. A.P. Sasmito, J.C. Kurnia, A.S. Mujumdar, Numerical evaluation of laminar heat transfer enhancement in nanofluid flow in coiled square tubes. Nanoscale Res. Lett. 6, 376 (2011). https://doi.org/10.1186/155 6-276X-6-376

64. F. Arpino, G. Cortellessa, A. Mauro, Transient thermal analysis of natural convection in porous and partially porous cavities. Numer. Heat Transf. Part A Appl. 67, 605-631 (2014). https://doi.org/10.1080/ 10407782.2014.949133

65. M. Yadegari, A.B. Khoshnevis, Numerical study of the effects of adverse pressure gradient parameter, turning angle and curvature ratio on turbulent flow in 3D turning curved rectangular diffusers using entropy generation analysis. Eur. Phys. J. Plus 135, 548 (2020). https://doi.org/10.1140/epjp/s13360-02 0-00561-y 